\title{
Elastic Analysis of Steel Beams Strengthened with GFRP Plates Including Preexisting Loading Effects
}

\author{
Phe Van Pham, S.M.ASCE${ }^{1}$; Magdi Mohareb, M.ASCE${ }^{2}$; and Amir Fam, M.ASCE ${ }^{3}$ \\ ${ }^{1}$ Doctoral Student, Department of Civil Engineering, University of Ottawa, Ottawa, ON, K1N6N5, Email: \\ ppham040@uottawa.ca \\ ${ }^{2}$ Professor, Department of Civil Engineering, University of Ottawa, Ottawa, ON, K1N6N5, Email: \\ mmohareb@uottawa.ca \\ ${ }^{3}$ Donald and Sarah Munro Chair Professor in Engineering and Applied Science, Department of Civil Engineering, \\ Queen's University, Kingston, ON, Canada K7L 3N6, Email: fam@civil.queensu.ca
}

\section{Abstract}

The present study develops a theory for the elastic analysis of a pre-loaded wide flange steel beam, strengthened with two Glass Fiber Reinforced Polymer (GFRP) plates bonded to both flanges, then subjected to additional loads. Starting with the principle of stationary potential energy, the governing equilibrium equations and corresponding boundary conditions are formulated prior to and after GFRP strengthening. The resulting theory involves four coupled equilibrium equations and 10 boundary conditions. A general closed form solution is then provided for general loading and boundary conditions. Detailed comparisons with three-dimensional finite element solutions show that the theory provides reliable predictions for the displacements and stresses. A parametric study is then developed to quantify the effects of strengthening, GFRP plate thicknesses, and preexisting loads, on the capacity of the strengthened beam.

Key words: steel beam, GFRP, strengthening, closed form solution, loading history

This article is to be cited as

Pham PV, Mohareb M, Fam A, (2017) Elastic Analysis of Steel Beams Strengthened with GFRP Plates Including Preexisting Loading Effects, Journal of Structural Engineering, 143(12): 04017163

The copy-edited version of this article is available at https://doi.org/10.1061/(ASCE)ST.1943-541X.0001904

This material may be downloaded for personal use only. Any other use requires prior permission of the American Society of Civil Engineers 


\section{INTRODUCTION AND BACKGROUND}

Strengthening steel structures using adhesively bonded FRP plates has been extensively studied in recent years due to the advantages this method offers; primarily the ease and speed of installation, and lightweight, compared to welded or bolted steel plates. The majority of studies focused on the use of carbon-FRP (CFRP) plates because of their higher Young modulus which can approach or exceed that of steel (Miller et al. 2001, Zhao and Zhang 2007, Harris and El-Tawil 2008, and Fam et al. 2009). GFRP plates, on the other hand, are considerably lower in cost than CFRP plates and their lower elasticity modulus can be compensated for by the fact that GFRP plates are typically thicker (El Damatty and Abushagur 2003 and El Damatty et al. 2003) than CFRP sheets. Thick GFRP plates with low elasticity modulus typically offer a higher flexural stiffness compared to thin CFRP plates and thus can be advantageous in strengthening thin compression flanges against local buckling (Aguilera and Fam 2013). Additionally, when in contact with steel, GFRP do not induce galvanic corrosion.

The beneficial effect of strengthening the tensile flanges of the $\mathrm{W}$-steel beam sections is widely reported in literature. Siddique and El Damatty (2013) reported a load capacity increase of 15\% whereas deflection at failure increased by $99 \%$ for cantilevers. The beneficial effect of GFRP was observed in experiments by El Damatty and Abushagur (2003), Holloway et al. (2006), Teng and Hu (2007), Correia et al. (2011), Siddique and Damatty (2012, 2013), Aguilera and Fam (2013), and Torabizaheh (2013). By using a single 19mm thick-GFRP plate on the tension side of a W150x13 cantilever, Pham and Mohareb (2015b) predicted a reduction in deflection and stresses of about $29 \%$ and $11 \%$, respectively. 
As indicated, GFRP plates can be relatively thick and are thus potentially effective on the compression side of a steel beam. Accord and Earl (2006) used four 6.35mm-thick GFRP plates to strengthen the compressive flange of a W-steel beam. The GFRP plates had an elasticity modulus in compression of 27.6 GPa. El Damatty and Abushagur (2003) tested 19mm-thick GFRP plates in shear lap tests. The plates had a compressive strength of $207 \mathrm{MPa}$ and a modulus of elasticity in compression of $17 \mathrm{GPa}$.

Strengthening of the compression zone of steel beams using GFRP plates was also reported in Westover (1998), (Correia et al. 2011), and Elchalakani and Fernando (2012). Compressive failures of GFRP plates were observed to be associated with layer delamination (Westover, 1998). Correia et al. (2011) provided a review of the compressive properties of pultruded GFRP composites and indicated that the compressive strength ranges from $20 \%$ to $80 \%$ of the tensile strength. Also, in Correia et al. (2011), the GFRP modulus of elasticity in compression was reported to be $80 \%$ of the tensile modulus.

A few studies analyzed beams strengthened with GFRP plates (El Damatty and Abushagur 2003, Linghoff et al. 2010a, 2010b, and Pham and Mohareb 2014, 2015a, 2015b). These studies focus on the response of steel beams strengthened with a single GFRP plate, either on the tensile or the compressive side. Another common theme among the above studies is fact that they do not capture the loading history nor do they capture initial stresses that may exist in the beam prior to and during strengthening.

In some beam strengthening applications, it is possible to fully unload an existing beam before retrofitting. In other cases, the existing loads cannot be fully removed, i.e., initial stresses and strains may exist in the steel beam at the time of strengthening. The effect of preloading on the strength of the retrofitted beam has been experimentally investigated for concrete beams 
strengthened with CFRP (Bonacci and Maalej 2000, Wenwei and Guo 2006, Wu et al. 2007, Kim and Shin 2011, and Richardson and Fam 2014). In some cases, the presence of preloading was shown to lower the capacity of the strengthened beam. Experimental investigations of preloaded I-section steel beams were also reported by Liu and Gannon (2009) and Qing et al. (2015) and showed a reduction in strength.

Analytical models for concrete beams strengthened with FRP plates which incorporate the effect of initial stresses/strains were reported in Wenwei and Guo (2006) and GangaRao et al. (2007). However, both studies adopted the transformed section method and are applicable for the case of full interaction. In the present study, given the large difference between the elastic moduli of the adhesive, GFRP and steel, only partial interaction is typically achieved and the transformed section solutions typically overestimates the strength and stiffness of the composite system. Ghafoori and Motavalli (2013) developed analytical models for the analysis of wide flange steel beams strengthened by a single pre-tensioned CFRP plate at the tensile flange, but did not capture the effect of preloading in the steel beam.

When both sides of a steel beam are accessible, as may be the case in open industrial structures or pipe racks, it may be beneficial to strengthen steel beams by bonding GFRP plates to both flanges (e.g., Youssef 2006, Shaat and Fam 2009, Elchalakani and Fernando 2012, Quin et al. 2015). For situations where only a single flange is accessible from the outside, the present model is able to determine the response of the beam by assigning low thicknesses and elastic constants to the absent GFRP and adhesive layer.

To the author's knowledge, no model is available for predicting the response of such systems. The present study aims to fill this gap by developing a theory for steel beams strengthened by two GFRP plates. The theory also captures the effects of partial interaction and pre-existing loads that 
may exist at the time of strengthening. The analysis is restricted to the elastic response of the strengthened system. As such, failure modes, whether by steel yielding, local buckling, GFRP plate through-thickness delamination, or plate de-bonding from steel, are beyond the scope of the present work.

When such modes are not critical, the present solution is expected to provide means to quantify the capacity of beams with non-compact (class 3) cross-sections strengthened with GFRP plates. When used for compact sections (classes 1 and 2), the theory is expected to predict only a conservative lower bound of the strength since it does not account for material plastic effects. From a serviceability viewpoint, the present model is expected to predict deflections at service load levels for beams with compact and non-compact sections as the strengthened system is expected to deform within the elastic range.

\section{SEQUENCE OF LOADING AND STRENGTHENING}

The loading and strengthening history for a steel beam are shown in Fig. 1a through configurations 1 to 6. Four deformation steps A-D are identified:

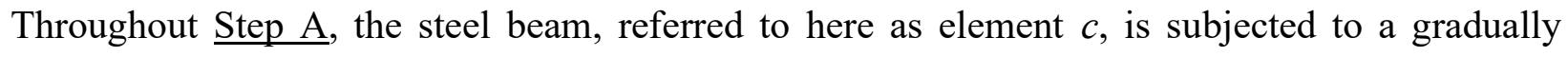
changing load from $q_{c, 1}(z)=0$ to $q_{c, 2}(z)$, where $z$ is a longitudinal coordinate along the beam axis. Under the applied load, the beam deforms from Configuration 1 and attains equilibrium at Configuration 2 through a transverse deflection $v_{2}(z)$.

Throughout Step B (from Configurations 2 to 4), two longitudinally-straight GFRP plates $a$ and $e$ are added for strengthening (Configuration 3). The difference in curvature between the straight GFRP plates and bent steel beam means that they are not in full contact along the span of the beam, causing gaps $\Delta_{a}(z)$ and $\Delta_{e}(z)$. To eliminate these gaps, adhesive layers $b$ and $d$ are first applied, 
and plates $a$ and $e$ are strapped to beam $c$ and are thus forced to bend through additional temporarily loads $q_{a, 4}(z)$ and $q_{e, 4}(z)$ (Configuration 4 and Fig. 1b). Loads $q_{a, 4}(z)$ and $q_{e, 4}(z)$ are intended to close the gaps $\Delta_{a}(z)$ and $\Delta_{e}(z)$, and are kept until bonding is fully developed between the steel flanges and GFRP.

Throughout $\underline{\text { Step C }}$ (from Configurations 4 to 5), external loads $q_{a, 4}(z)$ and $q_{e, 4}(z)$ due to strapping forces are removed (i.e., $q_{a, 5}(z)=q_{e, 5}(z)=0$ ) while the original external load acting on the steel beam is assumed to remain (i.e., $q_{c, 5}(z)=q_{c, 2}(z)$ ). The composite system then moves to a new equilibrium position (Configuration 5) characterized by four displacement fields; namely the total longitudinal displacement at the centroid of the top GFRP plate $w_{a, 5}(z)$, that of the steel beam centroid $w_{c, 5}(z)$, that of the bottom GFRP plate centroid $w_{e, 5}(z)$ and the transverse deflection $v_{5}(z)$ of the system, which is assumed equal to that of the steel beam (Fig. 2 where step $i$ is set to 5). In the general case where the GFRP plates have different geometries and/or material properties, the internal axial forces in both plates have different magnitudes and an internal force must be induced in the steel beam to enforce the internal axial force equilibrium condition, and hence the presence of displacement $w_{c, 5}(z)$. Because the adhesive layers provide partial interaction between the GFRP plates and the steel beam, a section initially plane for the composite system exhibits a kink at the adhesive locations.

In $\underline{\text { Step D }}$ (from Configurations 5 to 6), an additional operating external load $q_{6}(z)$ is applied to the strengthened beam. Under the new load, the composite beam attains equilibrium at Configuration 6 through total displacements $w_{a, 6}(z), w_{c, 6}(z), w_{e, 6}(z)$ and $v_{6}(z)$ (Fig. 2 in which 
the configuration $i=5$ and configuration $j=i+1=6)$. The loads and displacements corresponding to each configuration are summarized in Table 1.

\section{GENERAL MODEL AND SPECIAL CASES}

The aim is to develop a generic model to trace the entire equilibrium path of the loading and strengthening history described in the previous section. At a given equilibrium point $i$ of the trajectory 1-2-3-4-5-6, the composite system is assumed to be in equilibrium under a transverse $\operatorname{load} \bar{q}_{i}(z)$. The equilibrium configuration for the system (denoted as Configuration $i$ ) is assumed to be known and fully characterized by the known displacement fields $\bar{w}_{a, i}(z), \bar{w}_{c, i}(z), \bar{w}_{e, i}(z)$, $\bar{v}_{i}(z)$ (Fig. 2a). The system is then assumed to be subjected to an additional transverse load $\bar{q}_{j}(z)$ - Under the new load, the system reaches a new equilibrium configuration (denoted as Configuration $j=i+1)$. The system is assumed to undergo additional displacements $\bar{w}_{a, j}(z)$, $\bar{w}_{c, j}(z), \bar{w}_{e, j}(z), \bar{v}_{j}(z)$ (Fig. 2). Given $\bar{q}_{i}(z), \bar{q}_{j}(z), \bar{w}_{a, i}(z), \bar{w}_{c, i}(z), \bar{w}_{e, i}(z)$, and $\bar{v}_{i}(z)$, it is required to determine the $\bar{w}_{a, j}(z), \bar{w}_{c, j}(z), \bar{w}_{e, j}(z), \bar{v}_{j}(z)$

The deformations of step A can be obtained as special case of the general model by entirely eliminating the GFRP plates and adhesive layers from the composite section and setting loads $\bar{q}_{i}=q_{c, 1}=0$ and $\bar{q}_{j}=q_{c, 2}$. Displacement fields $\bar{w}_{c, i}(z)$ and $\bar{v}_{i}(z)$ at Configuration $i$ are set to zero, while displacement fields $\bar{w}_{c, j}(z)$ and $\bar{v}_{j}(z)$ at Configuration $j$ are set to 0 and $v_{2}$, respectively. Also, the deformations of step B can be obtained from the general model by entirely eliminating the adhesive layers (i.e., GFRP plates and W-beam works independently) and setting loads $\bar{q}_{i}=q_{c, 2}$ . Also, because the transverse displacements of both GFRP plates and W-beam are assumed equal, 
load potential energy gains of forces $q_{a, 4}$ and $q_{e, 4}$ are assumed to undergo the same transverse displacement as that of $q_{c, 2}$ and thus $\bar{q}_{j}=q_{a, 4}+q_{e, 4}$. Longitudinal displacement fields $\bar{w}_{a, i}(z)$, $\bar{w}_{c, i}(z), \bar{w}_{e, i}(z), \bar{w}_{a, j}(z), \bar{w}_{c, j}(z), \bar{w}_{e, j}(z)$ at both Configurations $i$ and $j$ are set to be zero, while transverse displacement fields $\bar{v}_{i}(z)$ and $\bar{v}_{j}(z)$ are set to be $v_{2}$.

Also, from the general model, by eliminating adhesive layers in only Configuration $i$ and setting loads $\bar{q}_{i}=q_{a, 4}+q_{c, 4}+q_{e, 4}$ and $\bar{q}_{j}=-q_{a, 4}-q_{e, 4}$, the displacements throughout Step C can be recovered. Longitudinal displacements $\bar{w}_{a, i}(z), \bar{w}_{c, i}(z)$ and $\bar{w}_{e, i}(z)$ in Configuration $i$ are set to zero while transverse displacement $\bar{v}_{i}(z)$ is set to $v_{2}$. Also, longitudinal displacements $\bar{w}_{a, j}(z)$, $\bar{w}_{c, j}(z)$ and $\bar{w}_{e, j}(z)$ in Configuration $j$ are respectively set to $w_{a, 5}, w_{c, 5}$ and $w_{e, 5}$, while transverse displacement $\bar{v}_{j}(z)$ is set equal to $\left(v_{5}-v_{2}\right)$.

Finally, Step D can be considered as the general case when loads $\bar{q}_{i}=q_{c, 5}$ and $\bar{q}_{j}=q_{c, 6}$ are set. Also, displacement fields $\bar{w}_{a, i}(z), \bar{w}_{c, i}(z), \bar{w}_{e, i}(z)$ and $\bar{v}_{i}(z)$ in Configuration $i$ are respectively taken as $w_{a, 5}, w_{c, 5}, w_{e, 5}$ and $v_{5}$ while displacement fields $\bar{w}_{a, j}(z), \bar{w}_{c, j}(z), \bar{w}_{e, j}(z), \bar{v}_{j}(z)$ in Configuration $j$ are taken as $\left(w_{a, 6}-w_{a, 5}\right),\left(w_{c, 6}-w_{c, 5}\right),\left(w_{e, 6}-w_{e, 5}\right)$ and $\left(v_{6}-v_{5}\right)$.

\section{DIMENSIONS AND COORDINATES}

The dimensions of the five-layer cross-section are shown in Fig. 3. The thicknesses of GFRP plate $a$ and adhesive layer $b$ are $t_{a}$ and $t_{b}$ while their width $b_{a}$ is identical. Also, the thicknesses of GFRP plate $e$ and adhesive layer $d$ are $t_{e}$ and $t_{d}$, and their width $b_{e}$ is also identical. Wide flange beam $c$ has a depth $h_{c}$, a flange width $b_{c}$, a flange thickness $t_{c}$, and a web thickness $w_{c}$. A global 
right-hand coordinate system $O X Y Z$ is selected as shown. Local coordinates $\left(s_{k}, n_{k}\right)$ where $k=a, b, c, d, e$ are also selected for each layer in which $s_{k}$ is oriented in tangential direction to the contour, and $n_{k}$ is oriented in normal direction to the contour.

\section{ASSUMPTIONS}

The steel beam and GFRP plates are considered as three Gjelsvik beams (Gjelsvik 1981). For each of the two components, the following assumptions are made:

(i) The shear strain $\gamma_{s z}$ of the middle surface is assumed to vanish,

(ii) The middle surface contours of all three sections do not deform in their own plane,

(iii) Each component behaves as a thin shell, in line with the Kirchhoff assumption that straight lines remain normal to the middle surface during deformation,

(iv) Forces applied by the straps to bring the intially straight GFRP plates into contact with the curved steel beam are assumed to preserve the initial curvature of the steel beams.

The following additional assumptions are made regarding the adhesive material:

(v) Perfect bond is assumed at the adhesive-GFRP and adhesive-steel interfaces,

(vi) The adhesive is treated as an elastic material with a small modulus of elasticity compared to those of the steel and GFRP. Thus, adhesive normal stresses in the longitudinal direction are considered negligible compared to those of the GFRP and steel,

(vii) The thickness of the adhesive is assumed to remain constant throughout deformation,

(viii) Within the steel and GFRP, only the normal stresses in the longitudinal direction and the shear stresses in the tangential plane are assumed to contribute to the internal strain energy while contributions of other stresses are assumed to be comparatively negligible,

(ix) Displacement fields at a point within the adhesive, are linearly interpolated from those at the steel-adhesive and GFRP-adhesive interfaces. 
The following assumption is made regarding the constitutive behavior of the materials:

(x) Steel is assumed is assumed to remain in the elastic range. The model is not intended to capture the post-yield response of the steel. In a strict sense, GFRP can exhibit orthotropic properties. However, given that only the longitudinal normal stresses and the shear stresses in the tangential plane are assumed to contribute to the internal strain energy (assumption viii), its relevant constitutive properties are fully characterized through only two constitutive constants; the longitudinal elastic modulus and a single shear modulus, in a manner similar to linearly elastic isotropic material. Thus, it is common to treat GFRP and CFRP as an isotropic material (e.g., Miller et al. 2001, El Damatty and Abushagur 2003, and Deng et al. 2004).

The following assumption is made regarding the nature of analysis:

(xi) Geometric and material non-linear and inertial effects as well as failure modes including yielding, buckling, delamination, and de-bonding are beyond the scope of the model.

\section{FORMULATION}

\section{Kinematic Relations}

The transverse and longitudinal displacements of a generic point within each layer are respectively

denoted as $\bar{v}_{k}(z)$ and $\widetilde{w}_{k}^{*}(s, n, z)$. These displacements are expressed in terms of centroidal displacement fields, coordinate $y_{c}(s)$ and tangential angle $\alpha\left(s_{c}\right)$ to the section contour:

$$
\tilde{w}_{k}^{*}=\left\{\begin{array}{cc}
\bar{w}_{a, k}(z)-n_{a} \bar{v}_{k}^{\prime}(z) & \left(n_{a}, z\right) \in \Omega_{a} \\
\bar{w}_{c, k}(z)-\left[y\left(s_{c}\right)+n_{c} \cos \alpha\left(s_{c}\right)\right] \bar{v}_{k}^{\prime}(z) & \left(s_{c}, n_{c}, z\right) \in \Omega_{c} \\
\bar{w}_{e, k}(z)+n_{e} \bar{v}_{k}^{\prime}(z) & \left(n_{e}, z\right) \in \Omega_{e}
\end{array} \quad(k=i \text { or } j)\right.
$$


where $k$ denotes configurations " $i "$ or " $j "$ as defined in Fig. 2 and $\Omega_{l}$ denotes the volumes of components $l=a, c, e$. The longitudinal displacement within adhesive layers $b$ and $d$ are linearly interpolated from the displacements at the interfaces with GFRP and steel, i.e.,

$$
\left\{\begin{array}{l}
\tilde{w}_{k}^{*}\left(n_{b}, z\right) \\
\tilde{w}_{k}^{*}\left(n_{d}, z\right)
\end{array}\right\}=\left(\frac{1}{2}-\frac{n_{b}}{t_{b}}\right)\left\{\begin{array}{l}
\bar{w}_{a, k}(z)-\frac{t_{a}}{2} \bar{v}_{k}^{\prime}(z) \\
\bar{w}_{e, k}(z)+\frac{t_{e}}{2} \bar{v}_{k}^{\prime}(z)
\end{array}\right\}+\left(\frac{1}{2}+\frac{n_{b}}{t_{b}}\right)\left\{\begin{array}{l}
\bar{w}_{c, k}(z)+\frac{h_{c}}{2} \bar{v}_{k}^{\prime}(z) \\
\bar{w}_{c, k}(z)-\frac{h_{c}}{2} \bar{v}_{k}^{\prime}(z)
\end{array}\right\}
$$

Strain-displacement relations: Longitudinal strains $\varepsilon=\partial \widetilde{w}^{*} / \partial z$ is provided for each component as

$$
\varepsilon_{k}=\left\{\begin{array}{cc}
\bar{w}_{a, k}{ }^{\prime}(z)-n_{a} \bar{v}_{k}{ }^{\prime \prime}(z) & \left(n_{a}, z\right) \in \Omega_{a} \\
\bar{w}_{c, k}{ }^{\prime}(z)-\left[y\left(s_{c}\right)+n_{c} \cos \alpha\left(s_{c}\right)\right] \bar{v}_{k}{ }^{\prime \prime}(z) & \left(s_{c}, n_{c}, z\right) \in \Omega_{c} \\
\bar{w}_{e, k}{ }^{\prime}(z)+n_{e} \bar{v}_{k}{ }^{\prime \prime}(z) & \left(n_{e}, z\right) \in \Omega_{e}
\end{array}\right.
$$

Also, the transverse shear strains within adhesive layers is given by $\gamma=\partial \widetilde{w}^{*} / \partial n+\partial \bar{v} / \partial z$, i.e.,

$$
\begin{aligned}
& \gamma_{k}\left(n_{b}, z\right)=-\frac{1}{t_{b}} \bar{w}_{a, k}(z)+\frac{1}{t_{b}} \bar{w}_{c, k}(z)+\frac{t_{a}+h_{c}+2 t_{b}}{2 t_{b}} \bar{v}_{k}^{\prime}(z) ; \quad n_{b} \in \Omega_{b} \\
& \gamma_{k}\left(n_{d}, z\right)=-\frac{1}{t_{d}} \bar{w}_{e, k}(z)+\frac{1}{t_{d}} \bar{w}_{c, k}(z)-\frac{t_{e}+h_{c}-2 t_{d}}{2 t_{d}} \bar{v}_{k}^{\prime}(z) ; \quad n_{d} \in \Omega_{d}
\end{aligned}
$$

Stress-displacement relations: Assuming linear isotropic material responses, the longitudinal stresses are related to the longitudinal normal strain through:

$$
\sigma_{k}=\left\{\begin{array}{cc}
E_{a}\left[\bar{w}_{a, k}{ }^{\prime}(z)-n_{a} \bar{v}_{k}^{\prime \prime}(z)\right] & n_{a} \in \Omega_{a} \\
E_{c}\left[\bar{w}_{c, k}^{\prime}(z)-\left[y\left(s_{c}\right)+n_{c} \cos \alpha\left(s_{c}\right)\right] \bar{v}_{k}^{\prime \prime}(z)\right] & s_{c} \in \Omega_{c} \\
E_{e}\left[\bar{w}_{e, k}{ }^{\prime}(z)+n_{e} \bar{v}_{k}^{\prime \prime}(z)\right] & n_{e} \in \Omega_{e}
\end{array}\right.
$$

and shear stresses within the adhesive layers are: 


$$
\begin{aligned}
& \tau_{k}\left(n_{b}, z\right)=G_{b}\left[-\frac{1}{t_{b}} \bar{w}_{a, k}(z)+\frac{1}{t_{b}} \bar{w}_{c, k}(z)+\frac{t_{a}+h_{c}+2 t_{b}}{2 t_{b}} \bar{v}_{k}^{\prime}(z)\right] ; \quad n_{b} \in \Omega_{b} \\
& \tau_{k}\left(n_{d}, z\right)=G_{d}\left[-\frac{1}{t_{d}} \bar{w}_{e, k}(z)+\frac{1}{t_{d}} \bar{w}_{c, k}(z)-\frac{t_{e}+h_{c}-2 t_{d}}{2 t_{d}} \bar{v}_{k}^{\prime}(z)\right] ; \quad n_{d} \in \Omega_{d}
\end{aligned}
$$

where $E_{l}$ are the moduli of elasticity of layers $l=a, c, e$, and $G_{l}$ are shear moduli of layers $b, d$

\section{Total Potential Energy:}

Under pre-existing loads $\bar{q}_{i}(z)$, equilibrium configuration $i$, as characterized by displacement fields $\bar{w}_{a, i}(z), \bar{w}_{e, i}(z), \bar{v}_{i}(z)$, corresponding to the initial stresses $\bar{\sigma}_{i}$ and strains $\bar{\varepsilon}_{i}$, is assumed to be known. The system is then subjected to additional loads $\bar{q}_{j}(z)$. In going from configuration $i$ to $j$, the potential energy loss consists of two components: (1) Component $\bar{V}_{j}$ caused by load $\bar{q}_{i}(z)+\bar{q}_{j}(z)$ undergoing transverse displacement $\bar{v}_{j}(z)$, and (2) Components caused by axial forces $\bar{N}_{\alpha, i}\left(z_{0}\right)+\bar{N}_{\alpha, j}\left(z_{0}\right)$ undergoing displacement $\bar{w}_{\alpha, j}\left(z_{0}\right)$, shear forces $\bar{Q}_{\alpha, i}\left(z_{0}\right)+\bar{Q}_{\alpha, j}\left(z_{0}\right)$ undergoing displacement $\bar{v}_{j}\left(z_{0}\right)$, and bending moment $\bar{M}_{\alpha, i}\left(z_{0}\right)+\bar{M}_{\alpha, j}\left(z_{0}\right)$ undergoing rotations $\bar{v}_{j}^{\prime}\left(z_{0}\right)$, in which $z_{0}$ denotes the ends $z=0$ or $z=L$. Under the additional load $q_{j}(z)$, additional strains $\bar{\varepsilon}_{j}$ and stresses $\bar{\sigma}_{j}$ take place within the system. The total strain energy consists of two components; the first is induced by the initial stresses $\bar{\sigma}_{i}$ undergoing strains $\bar{\varepsilon}_{i}$ and is depicted by the rectangular area $A B C D$ (Fig.2b). This component gives rise to the internal strain energy terms $\left(\bar{U}_{a}, \bar{U}_{b}, \bar{U}_{c}, \bar{U}_{d}, \bar{U}_{e}\right)_{i, j}$. The second component is induced by stresses $\bar{\sigma}_{j}$ undergoing strains $\bar{\varepsilon}_{j}$ and is depicted by the triangular area $C D E$. This gives rise to the internal strain energy terms $\left(\bar{U}_{a}, \bar{U}_{b}, \bar{U}_{c}, \bar{U}_{d}, \bar{U}_{e}\right)_{j}$. As a result, the total potential energy can be expressed as: 


$$
\begin{aligned}
& \pi_{i j}=\left(\bar{U}_{a}+\bar{U}_{b}+\bar{U}_{c}+\bar{U}_{d}+\bar{U}_{e}\right)_{i, j}+\left(\bar{U}_{a}+\bar{U}_{b}+\bar{U}_{c}+\bar{U}_{d}+\bar{U}_{e}\right)_{j}-\bar{V}_{j} \\
& -\left.\left[\bar{N}_{a, i}(z)+\bar{N}_{a, j}(z)\right] \bar{w}_{a, j}(z)\right|_{0} ^{L}-\left.\left[\bar{Q}_{a, i}(z)+\bar{Q}_{a, j}(z)\right] \bar{v}_{j}(z)\right|_{0} ^{L}-\left.\left[\bar{M}_{a, i}(z)+\bar{M}_{a, j}(z)\right] \bar{v}_{j}^{\prime}(z)\right|_{0} ^{L} \\
& -\left.\left[\bar{N}_{c, i}(z)+\bar{N}_{c, j}(z)\right] \bar{w}_{c, j}(z)\right|_{0} ^{L}-\left.\left[\bar{Q}_{c, i}(z)+\bar{Q}_{c, j}(z)\right] \bar{v}_{j}(z)\right|_{0} ^{L}-\left.\left[\bar{M}_{c, i}(z)+\bar{M}_{c, j}(z)\right] \bar{v}_{j}^{\prime}(z)\right|_{0} ^{L} \\
& -\left.\left[\bar{N}_{e, i}(z)+\bar{N}_{e, j}(z)\right] \bar{w}_{e, j}(z)\right|_{0} ^{L}-\left.\left[\bar{Q}_{e, i}(z)+\bar{Q}_{e, j}(z)\right] \bar{v}_{j}(z)\right|_{0} ^{L}-\left.\left[\bar{M}_{e, i}(z)+\bar{M}_{e, j}(z)\right] \bar{v}_{j}^{\prime}(z)\right|_{0} ^{L}
\end{aligned}
$$

Equation (7) can be expressed in terms of stress and strain fields as:

$$
\begin{aligned}
\pi_{i j}= & \left(\int_{L} \int_{A_{a}} \bar{\sigma}_{i} \bar{\varepsilon}_{j} d A_{a} d z+\iint_{L} \bar{\tau}_{A_{b}} \bar{\gamma}_{j} d A_{b} d z+\int_{L} \int_{A_{c}} \bar{\sigma}_{i} \bar{\varepsilon}_{j} d A_{c} d z+\int_{L} \int_{A_{d}} \bar{\tau}_{i} \bar{\gamma}_{j} d A_{d} d z+\iint_{L} \bar{\sigma}_{A_{e}} \bar{\sigma}_{i} \bar{\varepsilon}_{j} d A_{e} d z\right)+ \\
& +\frac{1}{2}\left(\int_{L} \int_{A_{a}} \bar{\sigma}_{j} \bar{\varepsilon}_{j} d A_{a} d z+\iint_{L} \bar{\tau}_{A_{b}} \bar{\gamma}_{j} d A_{b} d z+\iint_{L} \bar{\sigma}_{c} \bar{\sigma}_{j} \bar{\varepsilon}_{j} d A_{c} d z+\iint_{L} \bar{\tau}_{A_{d}} \bar{\gamma}_{j} d A_{d} d z+\iint_{L} \bar{A}_{e} \bar{\sigma}_{j} \bar{\varepsilon}_{j} d A_{e} d z\right)+ \\
& -\int_{L}\left[\bar{q}_{i}(z)+\bar{q}_{j}(z)\right] v_{j}(z) d z-\left.\left[\bar{N}_{a, i}(z)+\bar{N}_{a, j}(z)\right] \bar{w}_{a, j}(z)\right|_{0} ^{L}-\left.\left[\bar{Q}_{a, i}(z)+\bar{Q}_{a, j}(z)\right] \bar{v}_{j}(z)\right|_{0} ^{L} \\
& -\left.\left[\bar{M}_{a, i}(z)+\bar{M}_{a, j}(z)\right] \bar{v}_{j}^{\prime}(z)\right|_{0} ^{L}-\left.\left[\bar{N}_{c, i}(z)+\bar{N}_{c, j}(z)\right] \bar{w}_{c, j}(z)\right|_{0} ^{L}-\left.\left[\bar{Q}_{c, i}(z)+\bar{Q}_{c, j}(z)\right] \bar{v}_{j}(z)\right|_{0} ^{L} \\
& -\left.\left[\bar{M}_{c, i}(z)+\bar{M}_{c, j}(z)\right] \bar{v}_{j}^{\prime}(z)\right|_{0} ^{L}-\left.\left[\bar{N}_{e, i}(z)+\bar{N}_{e, j}(z)\right] \bar{w}_{e, j}(z)\right|_{0} ^{L}-\left.\left[\bar{Q}_{e, i}(z)+\bar{Q}_{e, j}(z)\right] \bar{v}_{j}(z)\right|_{0} ^{L} \\
& -\left.\left[\bar{M}_{e, i}(z)+\bar{M}_{e, j}(z)\right] \bar{v}_{j}^{\prime}(z)\right|_{0} ^{L}
\end{aligned}
$$

in which the following energy contributions have been defined

$$
\begin{aligned}
\left(\bar{U}_{a}, \bar{U}_{b}, \bar{U}_{c}, \bar{U}_{d}, \bar{U}_{e}\right)_{i, j} & =\int_{L}\left(\int_{A_{a}} \bar{\sigma}_{i} \bar{\varepsilon}_{j} d A_{a}, \int_{A_{b}} \bar{\tau}_{i} \bar{\gamma}_{j} d A_{b}, \int_{A_{c}} \bar{\sigma}_{i} \bar{\varepsilon}_{j} d A_{c}, \int_{A_{d}} \bar{\tau}_{i} \bar{\gamma}_{j} d A_{d}, \int_{A_{e}} \bar{\sigma}_{i} \bar{\varepsilon}_{j} d A_{e}\right) d z \\
\left(\bar{U}_{a}, \bar{U}_{b}, \bar{U}_{c}, \bar{U}_{d}, \bar{U}_{e}\right)_{j} & =\frac{1}{2} \iint_{L}\left(\int_{A_{a}} \bar{\sigma}_{j} \bar{\varepsilon}_{j} d A_{a}, \int_{A_{b}} \bar{\tau}_{j} \bar{\gamma}_{j} d A_{b}, \int_{A_{c}} \bar{\sigma}_{j} \bar{\varepsilon}_{j} d A_{c}, \int_{A_{d}} \bar{\tau}_{j} \bar{\gamma}_{j} d A_{d}, \int_{A_{e}} \bar{\sigma}_{j} \bar{\varepsilon}_{j} d A_{e}\right) d z \\
\bar{V}_{j} & =\int_{L}\left[\bar{q}_{i}(z)+\bar{q}_{j}(z)\right] v_{j}(z) d z
\end{aligned}
$$

From Eqs. (3)-(4), by substituting into Eq.(9), the variation of the total potential energy can be expressed as: 


$$
\begin{aligned}
& \delta \pi=\int_{L}\left\langle\delta \boldsymbol{\Delta}_{j}^{\prime}(z)\right\rangle_{1 \times 4}^{\mathbf{T}}\left[\mathbf{H}_{1}\right]_{4 \times 4}\left\{\boldsymbol{\Delta}_{i}^{\prime}(z)\right\}_{4 \times 1} d z+\int_{L}\left\langle\delta \boldsymbol{\Delta}_{j}^{\prime}(z)\right\rangle_{1 \times 4}^{\mathbf{T}}\left[\mathbf{H}_{1}\right]_{4 \times 4}\left\{\boldsymbol{\Delta}_{j}^{\prime}(z)\right\}_{4 \times 1} d z+ \\
& +\int_{L}\left\langle\delta \boldsymbol{\Delta}_{j}(z)\right\rangle_{1 \times 4}^{\mathbf{T}}\left[\mathbf{H}_{2}\right]_{4 \times 4}\left\{\boldsymbol{\Delta}_{i}(z)\right\}_{4 \times 1} d z+\int_{L}\left\langle\delta \boldsymbol{\Delta}_{j}(z)\right\rangle_{1 \times 4}^{\mathbf{T}}\left[\mathbf{H}_{2}\right]_{4 \times 4}\left\{\boldsymbol{\Delta}_{j}(z)\right\}_{4 \times 1} d z \\
& -\left(\int_{L}\left[q_{i}(z)+q_{j}(z)\right] v_{j}(z) d z\right)-\left.\left[\bar{N}_{a, i}(z)+\bar{N}_{a, j}(z)\right] \bar{w}_{a, j}(z)\right|_{0} ^{L}-\left.\left[\bar{Q}_{a, i}(z)+\bar{Q}_{a, j}(z)\right] \bar{v}_{j}(z)\right|_{0} ^{L} \\
& -\left.\left[\bar{M}_{a, i}(z)+\bar{M}_{a, j}(z)\right] \bar{v}_{j}^{\prime}(z)\right|_{0} ^{L}-\left.\left[\bar{N}_{c, i}(z)+\bar{N}_{c, j}(z)\right] \bar{w}_{c, j}(z)\right|_{0} ^{L}-\left.\left[\bar{Q}_{c, i}(z)+\bar{Q}_{c, j}(z)\right] \bar{v}_{j}(z)\right|_{0} ^{L} \\
& -\left.\left[\bar{M}_{c, i}(z)+\bar{M}_{c, j}(z)\right] \bar{v}_{j}^{\prime}(z)\right|_{0} ^{L}-\left.\left[\bar{N}_{e, i}(z)+\bar{N}_{e, j}(z)\right] \bar{w}_{e, j}(z)\right|_{0} ^{L}-\left.\left[\bar{Q}_{e, i}(z)+\bar{Q}_{e, j}(z)\right] \bar{v}_{j}(z)\right|_{0} ^{L} \\
& -\left.\left[\bar{M}_{e, i}(z)+\bar{M}_{e, j}(z)\right] \bar{v}_{j}^{\prime}(z)\right|_{0} ^{L}
\end{aligned}
$$

in which

$$
\begin{aligned}
& \left\langle\boldsymbol{\Delta}_{j}(z)\right\rangle_{1 \times 4}^{\mathbf{T}}=\left\langle\bar{w}_{a, j}(z) \quad \bar{w}_{e, j}(z) \quad \bar{w}_{c, j} \quad \bar{v}_{j}^{\prime}(z)\right\rangle ;\left\langle\left\langle\boldsymbol{\Delta}_{i}(z)\right\rangle_{1 \times 4}^{\mathbf{T}}=\left\langle\bar{w}_{a, i}(z) \quad \bar{w}_{e, i}(z) \quad \bar{w}_{c, i} \quad \bar{v}_{i}^{\prime}(z)\right\rangle ;\right. \\
& {\left[\mathbf{H}_{1}\right]_{4 \times 4}=\left[\begin{array}{cccc}
E_{a} A_{a} & 0 & 0 & 0 \\
0 & E_{e} A_{e} & 0 & 0 \\
0 & 0 & E_{c} A_{c} & 0 \\
0 & 0 & 0 & \alpha_{1}
\end{array}\right]}
\end{aligned}
$$

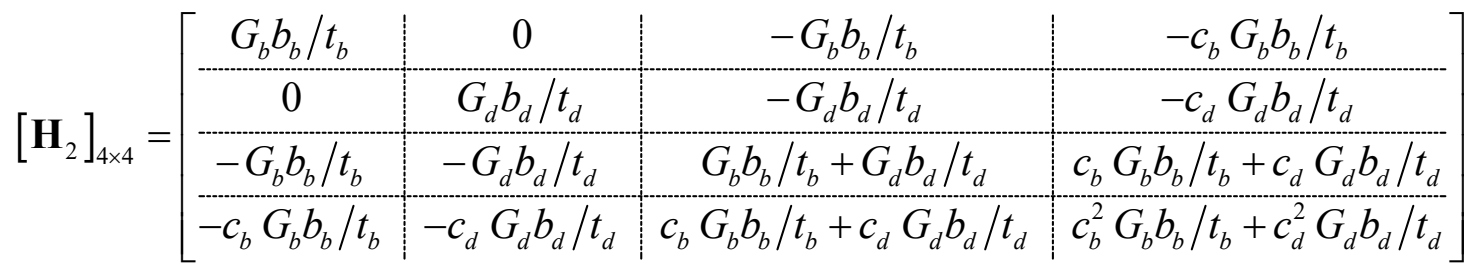

with $\alpha_{1}=E_{c} I_{x x c}+E_{a} I_{x x a}+E_{e} I_{x x e} ; \quad 2 c_{b}=2 t_{b}+h_{c}+t_{a} ; \quad 2 c_{d}=2 t_{d}-h_{c}-t_{e}$. 


\section{Equilibrium equations and boundary conditions:}

From Eq.(10), through integration by parts setting the variation of the potential energy to zero, one recovers the equilibrium equations. Expressed in a non-dimensional form, they take the form

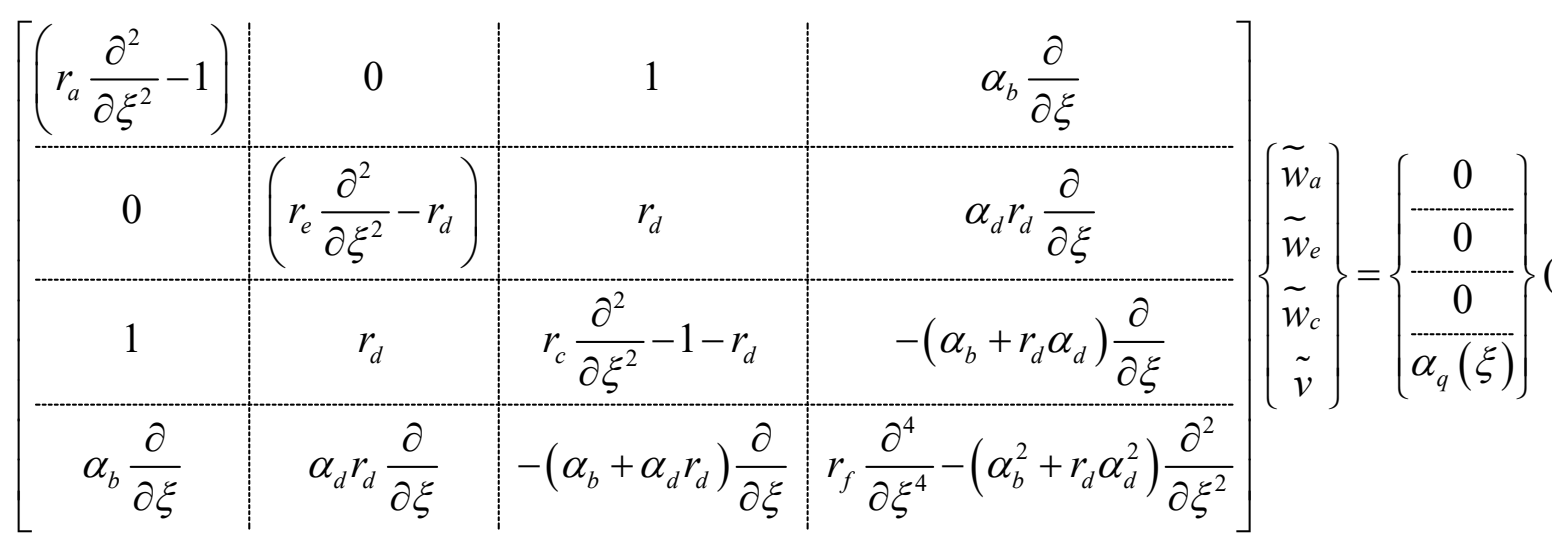

where $\xi=z / L$ is the non-dimensional longitudinal coordinate, $\widetilde{w}_{a}=\left(\bar{w}_{a, j}+\bar{w}_{a, i}\right) / L$ $\tilde{w}_{c}=\left(\bar{w}_{c, j}+\bar{w}_{c, i}\right) / L, \tilde{w}_{e}=\left(\bar{w}_{e, j}+\bar{w}_{e, i}\right) / L$, and $\tilde{v}=\left(\bar{v}_{j}+\bar{v}_{i}\right) / L$ are non-dimensional displacements, and following parameters have been introduced:

$$
\begin{aligned}
& r_{a}=\left(\frac{E_{a} A_{a}}{L^{2}}\right)\left(\frac{t_{b}}{G_{b} b_{b}}\right) ; \quad r_{e}=\left(\frac{E_{e} A_{e}}{L^{2}}\right)\left(\frac{t_{b}}{G_{b} b_{b}}\right) ; \quad r_{c}=\left(\frac{E_{c} A_{c}}{L^{2}}\right)\left(\frac{t_{b}}{G_{b} b_{b}}\right) ; \quad r_{d}=\left(\frac{G_{d} b_{d}}{t_{d}}\right)\left(\frac{t_{b}}{G_{b} b_{b}}\right) ; \\
& r_{f}=\frac{1}{L^{4}}\left(E_{c} I_{x x c}+E_{a} I_{x x a}+E_{e} I_{x x e}\right)\left(\frac{t_{b}}{G_{b} b_{b}}\right) ; \quad \alpha_{b}=\frac{2 t_{b}+h_{c}+t_{a}}{2 L} ; \quad \alpha_{d}=\frac{2 t_{d}-h_{c}-t_{e}}{2 L} ; \quad \alpha_{q}(\xi)=\frac{t_{b}\left(q_{i}+q_{j}\right)}{L G_{b} b_{b}} ;
\end{aligned}
$$

Ten boundary conditions arise from the boundary terms. These are: 


$$
\begin{aligned}
& \left.\delta \bar{w}_{a, j}(\xi)\left[r_{a} \tilde{w}_{a}^{\prime}(\xi)-\frac{t_{b}}{G_{b} b_{b} L^{2}}\left(\bar{N}_{a, i}(\xi)+\bar{N}_{a, j}(\xi)\right)\right]\right|_{0} ^{1}=0 \\
& \left.\delta \bar{w}_{e, j}(\xi)\left[r_{e} \tilde{w}_{e}^{\prime}(\xi)-\frac{t_{b}}{G_{b} b_{b} L^{2}}\left(\bar{N}_{e, i}(\xi)+\bar{N}_{e, j}(\xi)\right)\right]\right|_{0} ^{1}=0 \\
& \left.\delta \bar{w}_{c, j}(\xi)\left[r_{c} \tilde{w}_{c}^{\prime}(\xi)-\frac{t_{b}}{G_{b} b_{b} L^{2}}\left(\bar{N}_{c, i}(\xi)+\bar{N}_{c, j}(\xi)\right)\right]\right|_{0} ^{1}=0 \\
& \delta \bar{v}_{j}^{\prime}(\xi)\left[r_{f} \tilde{v}^{\prime \prime}(\xi)-\left.\frac{t_{b}}{G_{b} b_{b} L^{3}}\left[\bar{M}_{c, i}(\xi)+\bar{M}_{a, i}(\xi)+\bar{M}_{e, i}(\xi)+\bar{M}_{c, j}(\xi)+\bar{M}_{a, j}(\xi)+\bar{M}_{e, j}(\xi)\right]\right|_{0} ^{1}=0\right. \\
& -\left\{\begin{array}{l}
\delta \bar{v}_{1}(\xi)\left[r_{f} \tilde{v}^{\prime \prime \prime}(\xi)-\left(\alpha_{b}^{2}+r_{d} \alpha_{d}^{2}\right) \tilde{v}^{\prime}(\xi)+\alpha_{b} \widetilde{w}_{a}(\xi)+\alpha_{d} r_{d} \widetilde{w}_{e}(\xi)-\left(\alpha_{b}+\alpha_{d} r_{d}\right) \tilde{w}_{c}(\xi)\right)||_{0}^{1}=0 \\
\left.-\frac{t_{b}}{G_{b} b_{b} L^{2}}\left[\bar{Q}_{c, i}(\xi)+\bar{Q}_{a, i}(\xi)+\bar{Q}_{e, i}(\xi)+\bar{Q}_{c, j}(\xi)+\bar{Q}_{a, j}(\xi)+\bar{Q}_{e, j}(\xi)\right]\right]
\end{array}\right.
\end{aligned}
$$

\section{GENERAL SOLUTION}

The general solution is the sum of homogeneous and particular solutions, i.e.,

$$
\left\{\begin{array}{c}
\tilde{w}_{a}(\xi) \\
\tilde{w}_{e}(\xi) \\
\tilde{w}_{c}(\xi) \\
\tilde{v}(\xi)
\end{array}\right\}=\left\{\begin{array}{c}
\tilde{w}_{a}(\xi) \\
\tilde{w}_{e}(\xi) \\
\tilde{w}_{c}(\xi) \\
\tilde{v}(\xi)
\end{array}\right\}_{H}+\left\{\begin{array}{c}
\tilde{w}_{a}(\xi) \\
\tilde{w}_{e}(\xi) \\
\tilde{w}_{c}(\xi) \\
\tilde{v}(\xi)
\end{array}\right\}_{P}
$$

The homogeneous solution is recovered by setting the right hand side of Eqs. (11) to zero (Appendix 1), yielding

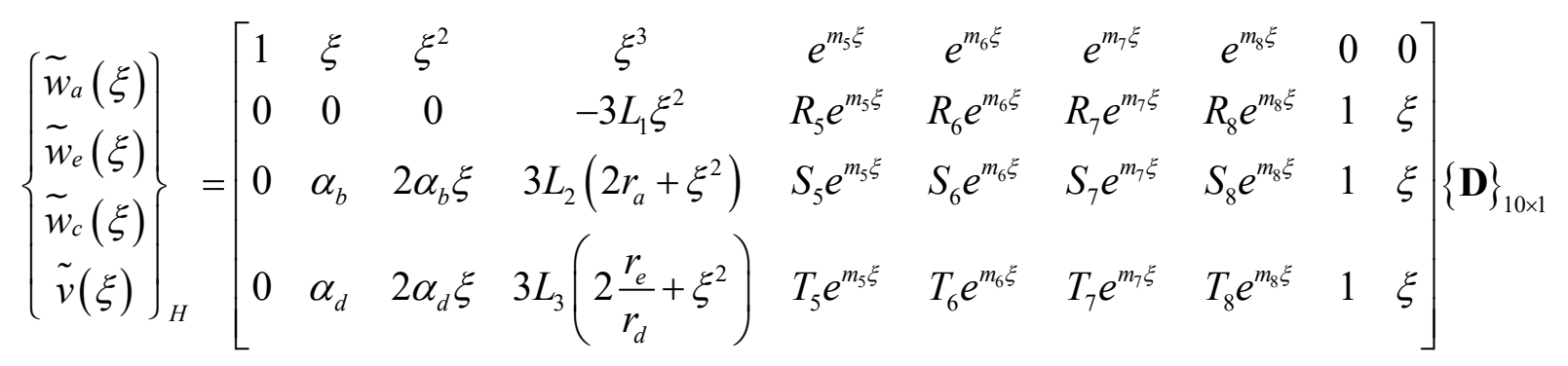


in which $\langle\mathbf{D}\rangle_{1 \times 10}^{\mathbf{T}}=\left\langle\begin{array}{llll}D_{1} & D_{2} & \ldots & D_{10}\end{array}\right\rangle$ is the vector of integration constants,

$$
\begin{aligned}
& L_{1}=\left(\alpha_{b} r_{a}+\alpha_{d} r_{e}\right) /\left(r_{a}+r_{e}+r_{c}\right), L_{2}=\left(\alpha_{b} r_{e}-\alpha_{d} r_{e}+\alpha_{b} r_{c}\right) /\left(r_{a}+r_{e}+r_{c}\right), \\
& L_{3}=\left(-\alpha_{b} r_{a}+\alpha_{d} r_{a}+\alpha_{d} r_{c}\right) /\left(r_{a}+r_{e}+r_{c}\right), \text { and } \\
& \begin{array}{l}
R_{k}=\frac{r_{a} r_{c} r_{e} r_{f}\left(\alpha_{b} r_{e}-\alpha_{d} r_{a} r_{d}\right)}{\left[\alpha_{b} \alpha_{d} r_{c}\left(r_{a} r_{d}-r_{e}\right)+r_{a} r_{e}\left(\alpha_{b}-\alpha_{d}\right)\left(\alpha_{b}+\alpha_{d} r_{d}\right)\right] r_{d}\left(r_{a}+r_{e}+r_{c}\right)} m_{k}^{5}-\frac{\left(\alpha_{b} r_{a}+\alpha_{d} r_{e}\right)}{\left(r_{a}+r_{e}+r_{c}\right)} m_{k} \\
\quad+\frac{\left(\alpha_{d} r_{a}^{2} r_{d}^{2}-\alpha_{b} r_{e}^{2}\right) r_{c} r_{f}+\left(\alpha_{b}+\alpha_{d} r_{d}\right)\left(r_{d} r_{a}-r_{e}\right) r_{a} r_{e} r_{f}+\left(\alpha_{d} r_{a} r_{d}-\alpha_{b} r_{e}\right)\left(\alpha_{b}^{2}+\alpha_{d}^{2} r_{d}\right) r_{a} r_{c} r_{e}}{\left[\alpha_{b} \alpha_{d} r_{c}\left(r_{a} r_{d}-r_{e}\right)+r_{a} r_{e}\left(\alpha_{b}-\alpha_{d}\right)\left(\alpha_{b}+\alpha_{d} r_{d}\right)\right] r_{d}\left(r_{a}+r_{e}+r_{c}\right)} m_{k}^{3} ; \\
S_{k}=R_{k}+\alpha_{b} m_{k}+\frac{r_{a}\left(\alpha_{b} r_{e}+\alpha_{d} r_{d} r_{e}+\alpha_{d} r_{c} r_{d}\right)}{\left(\alpha_{b} r_{e}-\alpha_{d} r_{a} r_{d}\right)} R_{k} m_{k}^{2}+\frac{\left(\alpha_{b}^{2} r_{a} r_{e}+\alpha_{d}^{2} r_{a} r_{d} r_{e}\right)}{\left(\alpha_{b} r_{e}-\alpha_{d} r_{a} r_{d}\right)} m_{k}^{3}-\frac{r_{a} r_{e} r_{f}}{\left(\alpha_{b} r_{e}-\alpha_{d} r_{a} r_{d}\right)} m_{k}^{5} ; \\
T_{k}=R_{k}+\alpha_{d} m_{k}-\frac{\alpha_{b} r_{c} r_{e}+\alpha_{b} r_{a} r_{e}+\alpha_{d} r_{a} r_{d} r_{e}}{r_{d}\left(\alpha_{b} r_{e}-\alpha_{d} r_{a} r_{d}\right)} R_{k} m_{k}^{2}-\frac{\alpha_{b}^{2} r_{a} r_{e}+\alpha_{d}^{2} r_{a} r_{d} r_{e}}{r_{d}\left(\alpha_{b} r_{e}-\alpha_{d} r_{a} r_{d}\right)} m_{k}^{3}+\frac{r_{a} r_{e} r_{f}}{r_{d}\left(\alpha_{b} r_{e}-\alpha_{d} r_{a} r_{d}\right)} m_{k}^{5} ; \\
k=5, \ldots, 8
\end{array}
\end{aligned}
$$

Parameters $m_{k}(k=5, \ldots, 8)$ appearing in Eq. (14) are four roots of the characteristic equation

$$
A m_{k}^{4}+B m_{k}^{2}+C=0 \text { where, }
$$

$$
\begin{aligned}
& A=\frac{r_{a} r_{c} r_{e} r_{f}\left(\alpha_{b} r_{e}-\alpha_{d} r_{a} r_{d}\right)}{\left\{\alpha_{b} \alpha_{d} r_{c}\left(r_{a} r_{d}-r_{e}\right)+r_{a} r_{e}\left(\alpha_{b}-\alpha_{d}\right)\left(\alpha_{b}+\alpha_{d} r_{d}\right)\right\} r_{d}\left(r_{a}+r_{e}+r_{c}\right)} ; \\
& B=\frac{\left(\alpha_{d} r_{a}^{2} r_{d}^{2}-\alpha_{b} r_{e}^{2}\right) r_{c} r_{f}+\left[\alpha_{d} r_{d}\left(r_{a} r_{d}+r_{a}+r_{c}\right)-\alpha_{b}\left(r_{e} r_{d}+r_{e}+r_{c} r_{d}\right)\right] r_{a} r_{e} r_{f}+\left(\alpha_{d} r_{a} r_{d}-\alpha_{b} r_{e}\right)\left(\alpha_{b}^{2}+\alpha_{d}^{2} r_{d}\right) r_{a} r_{c} r_{e}}{\left\{\alpha_{b} \alpha_{d} r_{c}\left(r_{a} r_{d}-r_{e}\right)+r_{a} r_{e}\left(\alpha_{b}-\alpha_{d}\right)\left(\alpha_{b}+\alpha_{d} r_{d}\right)\right\} r_{d}\left(r_{a}+r_{e}+r_{c}\right)} ; \\
& C=-\left[\frac{\left(\alpha_{b} r_{a}+\alpha_{d} r_{e}\right)}{\left(r_{a}+r_{e}+r_{c}\right)}+\frac{\left(\alpha_{d} r_{a} r_{d}-\alpha_{b} r_{e}\right) r_{f}+r_{a} r_{e}\left(\alpha_{d}-\alpha_{b}\right)\left(\alpha_{b}^{2}+\alpha_{d}^{2} r_{d}\right)}{\alpha_{b} \alpha_{d} r_{c}\left(r_{a} r_{d}-r_{e}\right)+r_{a} r_{e}\left(\alpha_{b}-\alpha_{d}\right)\left(\alpha_{b}+\alpha_{d} r_{d}\right)}\right] ;
\end{aligned}
$$

For the particular solution, it is expedient to expand the load function $\alpha_{q}(\xi)$, in Eqs. (11) using a

Fourier series decomposition in the domain $0 \leq \xi \leq 1$, yielding

$$
\alpha_{q}(\xi)=\sum_{n=1}^{n=n_{\max }} \alpha_{n q} \sin (n \pi \xi)
$$


where $n$ is a positive integer ranging from $n=1,2 \ldots n_{\max }$. In theory, an exact solution is obtained when $n_{\max } \rightarrow \infty$ but practically, convergence is attained by taking a finite number of terms. Also, $\alpha_{n q}$ is defined as

$$
\alpha_{n q}=2 \int_{0}^{1} \alpha_{q}(\xi) \sin (n \pi \xi) d \xi
$$

The particular solution is then assumed to take the form

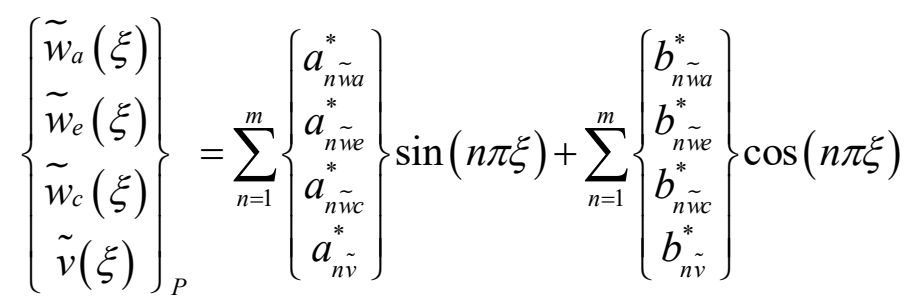

\begin{tabular}{|c|c|c|c|c|c|c|c|c|c|}
\hline$\eta_{1}(n)$ & 0 & 0 & 0 & 1 & 0 & 0 & $-\eta_{2}(n)$ & $a_{n \tilde{\text { u }}}^{*}$ & 0 \\
\hline 0 & $\eta_{1}(n)$ & 0 & 0 & 0 & 1 & $\eta_{2}(n)$ & 0 & $b_{n \tilde{\text { พิa }}}^{*}$ & 0 \\
\hline 0 & 0 & $\eta_{3}(n)$ & 0 & $r_{d}$ & 0 & 0 & $-\eta_{4}(n)$ & $a_{n \text { พैe }}^{*}$ & 0 \\
\hline 0 & 0 & 0 & $\eta_{3}(n)$ & 0 & $r_{d}$ & $\eta_{4}(n)$ & 0 & & 0 \\
\hline 1 & 0 & $r_{d}$ & 0 & $\eta_{5}(n)$ & 0 & 0 & $\eta_{6}(n)$ & $\overline{a_{n \tilde{\mu}}^{*}}$ & 0 \\
\hline 0 & 1 & 0 & $r_{d}$ & 0 & $\eta_{5}(n)$ & $-\eta_{6}(n)$ & 0 & $b_{n \tilde{\omega} c}^{*}$ & 0 \\
\hline 0 & $-\eta_{2}(n)$ & 0 & $-\eta_{4}(n)$ & 0 & $\eta_{6}(n)$ & $\eta_{7}(n)$ & 0 & $u_{n v}$ & $\overline{\alpha_{n q}}$ \\
\hline$\eta_{2}(n)$ & 0 & $\eta_{4}(n)$ & 0 & $-\eta_{6}(n)$ & 0 & 0 & $\eta_{7}(n)$ & $h^{*}$ & 0 \\
\hline
\end{tabular}

From Eqs. (17), by substituting into Eqs. (11) and noting that $\xi$ is arbitrary, one obtains

where $\eta_{1}(n)=-\left[r_{a}(n \pi)^{2}+1\right] ; \eta_{2}(n)=\alpha_{b}(n \pi) ; \eta_{3}(n)=-\left[r_{e}(n \pi)^{2}+r_{d}\right] ; \eta_{4}(n)=\alpha_{d} r_{d}(n \pi)$; $\eta_{5}(n)=-\left[r_{c}(n \pi)^{2}+1+r_{d}\right] ; \eta_{6}(n)=(n \pi)\left(\alpha_{b}+r_{d} \alpha_{d}\right) ; \eta_{7}(n)=r_{f}(n \pi)^{4}+\left(\alpha_{b}^{2}+r_{d} \alpha_{d}^{2}\right)(n \pi)^{2}$.

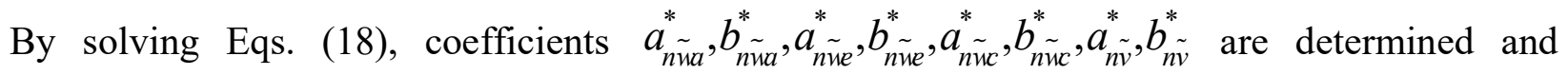
substituted into Eqs. (17) to yield the particular solution. 


\section{MODEL VERIFICATION}

The validity of the results based on the present formulation will be assessed through comparison with Finite Element Analysis (FEA) using the ABAQUS program. Also, 3D analyses based on the C3D8R element within the ABAQUS library will be used for verification. The C3D8R element is a 3D eight-node brick element with reduced integration. A mesh sensitivity study was conducted for similar problems and the details and specifics of the converged mesh have been reported in (Pham and Mohareb 2015b).

A $3.0 \mathrm{~m}$ span simply supported beam consists of a W150x13 steel beam (flange width $=100 \mathrm{~mm}$, flange thickness $=4.9 \mathrm{~mm}$, depth $=148 \mathrm{~mm}$ and web thickness $=4.3 \mathrm{~mm}$ ) is preloaded by a transversely uniform line load $q_{c, 2}=6.0 \mathrm{kN} / \mathrm{m}$ acting at the steel section centroidal axis (Step B).

Strengthening is contemplated by bonding two originally straight GFRP plates to the top and bottom flanges of the steel beam over the whole span (Step C). Both GFRP plates have identical thicknesses and widths $\left(t_{a}=t_{e}=19 \mathrm{~mm}, b_{a}=b_{e}=100 \mathrm{~mm}\right)$ and are bonded to the steel beam through two identical adhesive layers with the thickness $t_{b}=t_{d}=1.0 \mathrm{~mm}$ (Fig. 3). Steel modulus of elasticity is taken as $200 \mathrm{GPa}$, that of GFRP is assumed as $E_{a}=E_{e}=42 \mathrm{GPa}$, and the shear modulus of the adhesive is $G_{b}=G_{d}=0.4 \mathrm{GPa}$. Poisson's ratio $\mu$ for all three materials is taken as 0.3 . The yielding strength of steel is $350 \mathrm{MPa}$, the rupture strength of GFRP plates is $896 \mathrm{MPa}$, and the shear strength of adhesive is $9.0 \mathrm{MPa}$.

After strengthening, the composite beam is subjected to additional uniform transverse line load $q_{c, 6}=6.0 \mathrm{kN} / \mathrm{m}$ (Step D). It is required to compare (i) the transverse displacement and maximum longitudinal normal stresses for the wide flange beam and GFRP plates, and (ii) the transverse 
shear stresses within the adhesive layers as predicted by the present closed form solution and the 3D FEA under ABAQUS.

The applicable boundary conditions are $\widetilde{w}_{a}^{\prime}(0)=\widetilde{w}_{e}^{\prime}(0)=\widetilde{w}_{c}^{\prime}(0)=\bar{v}_{j}(0)=\tilde{v}^{\prime \prime}(0)=\widetilde{w}_{a}^{\prime}(1)$ $=\widetilde{w}_{e}^{\prime}(1)=\bar{w}_{c, j}(1)=\bar{v}_{j}(1)=\tilde{v}^{\prime \prime}(1)=0$. A mesh study indicated that convergence is attained when $n=10$ is taken. Also, a mesh sensitivity study on the 3D FEA model indicated that convergence is attained by taking 20 elements along each flange overhang, four elements across the flange thickness, 70 elements along the web height, four elements across the web thickness, four elements across each adhesive thickness, eight elements across each GFRP thickness, and 750 elements in the longitudinal direction. In step A, the steel beam is first activated and bent while GFRP plates are deactivated. Then, in step B, GFRP plates are activated and prescribed to take the curvature of the farthermost fibers of the steel beam (i.e., top and bottom fibers). Also, adhesive layers are activated using the free strain option. In step C, the displacements applied to GFRP plates in step B are released. Finally, in step D, the steel is exposed to additional loading.

\section{Displacements:}

Figure 4a depicts the transverse displacements in Steps B and D. Maximum displacements at midspan in Step B and Step D obtained from present study are $5.3 \mathrm{~mm}$ and $8.1 \mathrm{~mm}$, respectively, while those predicted by the 3D FEA are $5.5 \mathrm{~mm}$ and $8.3 \mathrm{~mm}$, corresponding to $1.9 \%$ and $2.4 \%$ differences. The differences are attributed to neglecting the effect of transverse shear (Pham and Mohareb 2015b). The additional deflection in going from configuration 4 to 5 (Step C) is only $-0.011 \mathrm{~mm}$, corresponding to $0.1 \%$ of the displacement in Step D. This negligible displacement is attributed to the fact that loads $q_{a, 4}(z)$ and $q_{e, 4}(z)$ needed to bend the GFRP plates are rather small compared to $q_{c, 2}(z)$. 


\section{Longitudinal normal stresses:}

Figures $4 \mathrm{~b}$,c provide the maximum tensile stresses at the bottom fiber of the wide flange beam and at the bottom of GFRP plate $e$. Maximum stresses in Steps B and D in the steel provided by the present study are $83.7 \mathrm{MPa}$ and $127 \mathrm{MPa}$, respectively, and those provided by the 3D FEA model are $83.2 \mathrm{MPa}$ and $126.9 \mathrm{MPa}$, which correspond to $0.6 \%$ and $0.1 \%$ differences. Also, the maximum tensile stress induced in Step D in GFRP $a$ based on the present study is $13.54 \mathrm{MPa}$ while that based on the 3D-FEA is $13.55 \mathrm{MPa}$, a $0.1 \%$ difference. The additional longitudinal stress within the steel in Step C is only $-0.19 \mathrm{MPa}$ and is thus are negligible.

\section{Shear stresses in adhesive layers:}

Figure $4 \mathrm{~d}$ shows the 3D FEA transverse shear stresses $\tau_{n z}$ along the left edge of the beam and at the center line (both lines are shown on the plan view provided in Fig. 6d). The shear stress averaged over the width of the adhesive is also depicted. At $z=500 \mathrm{~mm}$, the maximum difference between the present solution and that based on 3D-FEA is $2.3 \%$. Near beam ends, i.e. at $z=30 \mathrm{~mm}$ , the 3D-FEA model predicts maximum shear stresses of $0.57 \mathrm{MPa}$ at the center fibers and peeling stresses of $0.13 \mathrm{MPa}$. Both stresses correspond to an effective Mises stress of $0.59 \mathrm{MPa}$ and are significantly less than the experimentally determined in El Damatty and Abushagur (2003). (In the later study, shear strength ranged from $20.9 \mathrm{MPa}$ to $34.3 \mathrm{MPa}$ while the peeling strength ranged from $0.95 \mathrm{MPa}$ to $6.01 \mathrm{MPa}$ ). Also, the strains predicted by the present model and ABAQUS were observed to be nearly constant across the adhesive depth in a manner consistent with Pham (2013).

Validation for different GFRP thicknesses: 
The validation is also extended for three pairs of GFRP plate thicknesses $\left(t_{a}, t_{e}\right)=(9,29),(19,19)$, and $(29,9) \mathrm{mm}$ (Table 2) while all other dimension parameters are kept identical. For all three cases, the transverse displacements in step C and D based on the present solution are observed to remain almost unchanged at $5.3 \mathrm{~mm}$ and $8.1 \mathrm{~mm}$, respectively, while those based on the 3D FEA solution were $5.5 \mathrm{~mm}$ and $8.3 \mathrm{~mm}$. The peak compressive and tensile stresses in the wide flange beam and GFRP plates are observed to change considerably (Table 2). The maximum difference between both solutions are $0.4 \%$ for the compression flange of the wide flange beam when $\left(t_{a}, t_{e}\right)=(29,9) \mathrm{mm}$ and $1.1 \%$ for GFRP plate $e$ when $\left(t_{a}, t_{e}\right)=(19,19) \mathrm{mm}$.

\section{Comparison with Transformed Section Method:}

The transformed section method is based on the assumption that plane section for the strengthened system remains plane throughout deformation. It is expected that plane section condition is approached when the elastic properties of the materials involved do not vary significantly. Depending on the type of adhesive selected, the elastic properties significantly vary at room temperature from as low as $0.7 \mathrm{MPa}$ for polyurethane (e.g., Huveners et al. 2007) to $3.5 \mathrm{GPa}$ for stiff epoxies (e.g., Hall, J. 2002). Further, for a given type of epoxy, the elastic properties have been reported to drop by orders of magnitudes when temperature rises from $20^{\circ} \mathrm{C}$ to $50^{\circ} \mathrm{C}$ (Sahin and Dawood 2016). It is thus of interest to quantify the adhesive shear modulus values needed to approach the plane section condition (i.e., full interaction).

Three adhesive shear moduli are selected for the comparison. These are Polyvinyl Butyral with $G_{d}=G_{d}=1.3 \mathrm{MPa}$ (Asik and Tezcan 2005), Cyanoacrylates with $G_{d}=G_{d}=0.4 \mathrm{GPa}$ (Hall 2002) 
and SP Spabond two part epoxy with $G_{d}=G_{d}=1.3 \mathrm{GPa}$ as estimated from the Young modulus and Poisson's ratio reported in (Dawood 2008).

For the two stiffer cases $G_{d}=G_{d}=0.4 G P a$ and $G_{d}=G_{d}=1.3 G P a$, the present model predicts identical deflections of $8.1 \mathrm{~mm}$ (Table 3). When the shear moduli are reduced to $G_{d}=G_{d}=1.3 \mathrm{MPa}$, the present model predicts a deflection of $8.9 \mathrm{~mm}$. Nearly identical predictions are obtained by ABAQUS in all cases. When adopting the transformed section method, the moment of inertia $I$ of composite section is found to be negligibly affected by the modulus of the adhesive. When $G_{d}=G_{d}=1.3 \mathrm{GPa}$, the moment of inertia is $I=1.1700 e+07 \mathrm{~mm}^{4}$ and when $G_{d}=G_{d}=0.4 \mathrm{GPa}$, the moment of inertia is $I=1.1693 e+07 \mathrm{~mm}^{4}$. These value compare to $I=1.1687 e+07 \mathrm{~mm}^{4}$ when $G_{d}=G_{d}=1.3 \mathrm{MPa}$, a mere $0.11 \%$ and $0.05 \%$ difference. Thus, the large difference in adhesive elasticity and shear moduli in all three cases results in essentially in the same predicted deflection of $8.0 \mathrm{~mm}$. For the cases of stiff adhesive shear moduli (i.e., 1.3 GPa and $0.4 \mathrm{GPa}$ ), the transformed section method under-predicts the deflection by $1.2 \%$. However, for the weak adhesive, the method is found to underpredict the displacement by $10.1 \%$. For the steel section, stress perdictions based on the transformed section agree with the present solution and $3 \mathrm{D}$ FEA within $0.4 \%$ for the stiff adhesives. However, the difference between predictions grows to $9.0 \%$ for the weak adhesive. For the GFRP, the present solution and ABAQUS predict a maximum normal stress of $13.5 \mathrm{MPa}$ in the case stiff adhesives. This compares to $11.4 \mathrm{MPa}$ as predicted by the transformed section method. In the present example, the transformed section method underpredicts the GFRP stresses by $15.5 \%$ difference for the case of stiff adhesive. The percentage difference improves to $5.6 \%$ for the case of the weak adhesive. Also, the present study 
and 3D FEA solutions show that for practical purposes the deflection and normal stresses in the steel and GFRP do not change for adhesive shear modulus values larger than 0.4GPa.

\section{EFFECTIVENESS OF STRENGTHENING}

As discussed in the previous example, the normal stresses in the GFRP plates and the shearing stresses in the adhesive layers were rather small compared to their respective material strengths. As a result, the effectiveness of strengthening can be assessed by comparing the peak displacements and normal stresses of the un-strengthened and strengthened steel beam. We recall that the beam was under a pre-existing load of $q_{c, 2}=6 \mathrm{kN} / \mathrm{m}$. The corresponding peak deflection and stresses are $5.5 \mathrm{~mm}$ and $84 \mathrm{MPa}$. If the un-strengthened beam is subjected to an additional $\operatorname{load} q_{c, 5}=6 \mathrm{kN} / \mathrm{m}$, the peak deflection would be $11 \mathrm{~mm}$ and the corresponding peak longitudinal normal stresses would increase to $168 \mathrm{MPa}$. If the beam is strengthened with FRP plates of equal thicknesses, the peak deflection is observed to drop by $27.3 \%$ to $8 \mathrm{~mm}$. The corresponding stress drops by $24.2 \%$ to $127 \mathrm{MPa}$. Also, as shown in Table 2, when the thickness of GFRP plates $a$ and $e$ are taken unequal, i.e., $\left.\left(t_{a}, t_{e}\right)=(29,9) \mathrm{mm}\right)$, the peak normal stresses are found to increase from $127 \mathrm{MPa}$ to $137 \mathrm{MPa}$, a $7.3 \%$ difference.

\section{PARAMETRIC STUDY}

\section{Effect of GFRP Elastic Modulus}

In the cases where the modulus of elasticity for both GFRP plates are taken equal in compression and tension, the previous section has shown that strengthening is most effective when both GFRP plate thicknesses are equal, resulting in the lowest normal stresses in the steel. This section explores the case where both GFRP plates have different elastic moduli as reported in Correia et 
al. (2011) where the GFRP compressive elastic modulus is reported to be $80 \%$ of that in tension. In such cases, the optimum thickness ratio $t_{a} / t_{e}$ needs to be established. Towards this goal, the simply supported composite beam as presented in Example 1 is re-considered, while changing the modulus of elasticity for GFRP plate $a$ from $42.0 \mathrm{GPa}$ to $33.6 \mathrm{GPa}$. The thickness of GFRP plate $a$ is assumed to range from $1.0 \mathrm{~mm}$ to approximately $30 \mathrm{~mm}$ while that of GFRP plate $e$ is varied from $37.0 \mathrm{~mm}$ to approximately $8.0 \mathrm{~mm}$ such that the total thickness $t_{a}+t_{e}=38 \mathrm{~mm}$, resulting in a constant volume of GFRP material in all cases. Figure 5 shows the deflection at mid-span and maximum longitudinal normal stresses at top and bottom fibers of the wide flange beam under the application of additional load $q_{c, 6}(z)$ in Step D versus the thickness ratio $t_{a} / t_{e}$. For $t_{a} / t_{e}=0.027$ , corresponding to $\left(t_{a}, t_{e}\right)=(1.0,37) \mathrm{mm}$, the deflection is $8.1 \mathrm{~mm}$. The deflection is observed to mildly increase to a peak value of $8.3 \mathrm{~mm}$ when $t_{a} / t_{e}=4$ corresponds to $\left(t_{a}, t_{e}\right)=(30.4,7.6) \mathrm{mm}$ . Based on a minimum deflection criterion, the solution $t_{a} / t_{e}=0.027$ or $\left(t_{a}, t_{e}\right)=(1.0,37) \mathrm{mm}$ provides the optimum design. Also shown are the peak compressive stresses at the top fiber of the steel section. The peak compressive stress is $149 \mathrm{MPa}$ and occurs at $t_{a} / t_{e}=0.027$ and is found to decrease as the ratio $t_{a} / t_{e}$ increases. A reverse trend is observed for the peak tensile stresses in the steel where they have a minimal value at stress of $t_{a} / t_{e}=0.027$ and monotonically increase to $140 M P a$ when $t_{a} / t_{e}=4$. The optimum $t_{a} / t_{e}$ ratio is that at which the peak tensile stress in the steel is equal to that of the peak compressive stresses. This condition is realized at a $t_{a} / t_{e}$ ratio of 1.22 . The corresponding deflection value is $8.2 \mathrm{~mm}$ which is marginally higher than the minimum deflection of $8.1 \mathrm{~mm}$ while the corresponding stress is $129 \mathrm{MPa}$. 


\section{Effect of GFRP Plate Thickness}

The previous case is revisited while keeping all parameters unchanged except that both GFRP plate thicknesses are varied. Unlike the previous case where the sum of plate thicknesses was kept constant, the present example investigates other cases where the sum of plate thicknesses is variable. Two thicknesses are considered for the GFRP plate: $t_{e}=19 \mathrm{~mm}$ and $t_{e}=30 \mathrm{~mm}$. For both cases, the thickness of GFRP plate $a$ is increased from $t_{a}=0.2 t_{e}$ to $t_{a}=4 t_{e}$. For $t_{e}=19 \mathrm{~mm}$, the mid-span deflection is observed to decrease from $8.9 \mathrm{~mm}$ at $t_{a}=0.2 t_{e}$ to $6.8 \mathrm{~mm}$ at $t_{a}=4 t_{e}$ (Fig. 6a). Also, for $t_{e}=30 \mathrm{~mm}$ the mid-span deflection decreases from $8.2 \mathrm{~mm}$ at $t_{a}=0.2 t_{e}$ to $6.1 \mathrm{~mm}$ at $t_{a}=4 t_{e}$ (Fig. 6b).

Also depicted in the figure are the peak compressive and tensile stresses in the steel. Of particular interest is to note that both curves intersect at a thickness ratio $t_{a} / t_{e}=1.22$ which exactly coincides with the optimum thickness ratio obtained in the previous case. However, unlike previous case, as $t_{a} / t_{e}$ exceeds 1.22 , both compressive and tensile stresses are observed to decrease. This is a natural outcome of the fact that thicknesses of both GFRP plates increase. As expected, the larger plate thicknesses are observed to correspond to a lower deflection.

\section{Effect of Pre-existing Load and Stresses}

The present example investigates the effect of initial stresses/strains state induced by the preexisting loads on the capacity of the strengthened beam. A 3m-span W150x13 cantilever steel beam is considered. All dimensions and material parameters are identical to those in Example 1. During strengthening, the beam is assumed to be under a pre-existing point load $P_{c, 2}$. The load can be acting downward (Fig. 7a.1) in cases where an existing structure cannot be fully unloaded prior to strengthening, or can vanish in cases where the structure is fully unloaded prior to strengthening 
(Fig. 7a.2). Alternatively, a hydraulic jack can be used to temporarily prop up the beam prior to and during strengthening (Fig. 7a.3) inducing a beneficial pre-stressing effect. For the problem under investigation, the load corresponding to the first yield of $\sigma_{y}=350 \mathrm{MPa}$ is $P_{c, 2}= \pm 9.4 \mathrm{kN}$ which defines the practical range of interest of pre-existing loading.

The total applied load versus the tip deflection is depicted in Fig. $7 \mathrm{~b}$ and the total applied load versus the peak normal stress is provided in Fig. 7c. Three cases corresponding to different preexisting load levels $P_{c, 2}=4.7 \mathrm{kN}$ (downward), $P_{c, 2}=0$ and $P_{c, 2}=-9.4 \mathrm{kN}$ (i.e., upward prestressing load) are depicted by the loading path 1-2a-6a,1-6b, and 1-2c-6c, respectively. Also, depicted as a reference is the loading path for the case for the un-strengthened beam. As marked on the figures, without strengthening, the beam is able to withstand a load of $9.4 \mathrm{kN}$, which corresponds to a peak stress of $350 M P a$ in the steel (Fig. 7c). When the beam is under a preexisting gravity load of $4.7 \mathrm{kN}$ during strengthening (Path 1-2a-6a), the strengthening allows the beam reach a load of $13.8 \mathrm{kN}$, a $47 \%$ increase over the un-strengthened case. If the beam is fully unloaded prior to strengthening (Path 1-6b), it can sustain a load of $18.1 \mathrm{kN}$, a 93\% increase over the strengthened case. The most beneficial strengthening scenario is the case where a pre-stressing load $P_{c, 2}$ is $=-9.4 k N$ (i.e. upward force) is applied. For this case, the beam is able to attain a gravity load of $26.8 \%$ which corresponds to $185 \%$ increase in capacity over the un-strengthened beam. The last scenario corresponds to the highest shear stresses in the adhesive and the highest normal stresses in the GFRP. Under this scenario, the stresses in the adhesive and GFRP are observed to remain significantly smaller than the respective material strengths. Thus, the capacity of the strengthened system solely depends on the peak stresses in the steel. Figure $7 \mathrm{~d}$ depicts the relationship between applied load $\left(P_{c, 2}+P_{c, 6}\right)$ the strengthened beam can withstand versus the peak 
stress in the steel. Also, shown on the top horizontal axis is the value of the pre-existing load $P_{c, 2}$. The total applied load $\left(P_{c, 2}+P_{c, 6}\right)$ is linearly related to the pre-existing load $P_{c, 2}$.

\section{Effect of Adhesive Shear Modulus}

In the previous example, the effect of the adhesive shear modulus on the total load is presented in Fig. 7d comparing the results for two shear moduli and $G_{b}=G_{d}=0.4 M P a$ (Cyanoacrylates, Polyurhetane) and $G_{b}=G_{d}=1.3 M P a$ (Polyvinyl Butyral). The slope of the total load to pre-stressing for the system with weaker shear modulus is milder than that with a stiffer adhesive, leading to a lower peak total load. At a pre-existing load $P_{c, 2}=-9.4 \mathrm{kN}$, a significant reduction of the adhesive shear modulus from $0.4 G P a$ to $1.3 \mathrm{MPa}$ causes a relatively mild reduction of the peak load reduction from $26.8 \mathrm{kN}$ to $21.7 \mathrm{kN}$

\section{SUMMARY AND CONCLUSIONS}

(1) A theory was developed for the analysis of preloaded/pre-stressed wide flange beams strengthened with GFRP plates bonded to both flanges through adhesive layers providing partial interaction. The theory results in four coupled differential equations of equilibrium and 10 boundary conditions.

(2) A closed form solution was developed for general loading and boundary conditions.

(3) For the examples investigated, the present theory provides stress and displacement predictions in the steel and GFRP in excellent agreement with those based on 3D FEA results. The maximum difference between both solutions is $2.4 \%$ for deflection $1.1 \%$ for stresses. For the adhesive, the shear stress obtained from the present study agrees well with the average shear stress obtained from 3D FEA results (i.e., the maximum difference between two solutions is about 2.3\%), except 
at the points of singularity, where the present theory, like other beam theories, does not capture localized stress concentrations.

(3) The present solution is computationally efficient when compared to ABAQUS 3D FEA solutions. For example, when the present analysis, when implemented under MATLAB R2011b, it was completed in 45 seconds on a computer with two Intel (Santa Clara, California) Xeon processors with an E5-24300 and a central processing unit with a speed of 2.20 and $64.0 \mathrm{~GB}$ of RAM. On the same computer, the run time of the 3D FEA ABAQUS model for the same problem, based on 2,243,000 C3H8R elements, was 5.43 hours.

(4) For the case where the elasticity moduli of both GFRP plates are identical, plates of equal thickness where found to optimize the design based on a stress in the steel criterion. For example, a 3m-span W130x15 simply supported beam strengthened with two $19 \mathrm{~mm}$ - thick GFRP plates has a peak normal stress that is $7.9 \%$ smaller than that in the wide flange beam strengthened with $9 m m$ and $29 m m$ - thick GFRP plates, of a similar total volume of GFRP.

(5) For the same problem, when the elasticity modulus of the compressive GFRP plate is taken as a $80 \%$ of that of the tensile GFRP plate, the most effective thickness ratio of the compressive plate to that of the tensile plate for strengthening is found to be 1.22 when the sum of the thicknesses of both GFRP plates is kept constant.

(6) Pre-existing loads acting on the beam during strengthening are shown to significantly affect the capacity of the system. Pre-stressing is found to be particularly beneficial in this respect.

(7) The examples investigated in the present study suggest that the strengthened system is only mildly sensitive to the shear modulus of the adhesive. 


\section{ACKNOWWLEGMENTS}

The authors gratefully acknowledge scholarship support from the University of Ottawa to the first

author and research funding from the Natural Sciences and Engineering Research Council (NSERC) of Canada to the second and third authors.

\section{APPENDIX 1: Homogeneous Solution of the Equilibrium Equations}

By expressing Eqs. (11) in an explicit form, introducing the non-dimensional coordinate $\xi=z / L$ and dividing all equation by $G_{b} b_{b} / t_{b}$, one obtains

$$
\begin{aligned}
& r_{a} D^{2} \widetilde{w}_{a}-\widetilde{w}_{a}+\widetilde{w}_{c}+\alpha_{b} D \tilde{v}=0 \\
& r_{e} D^{2} \widetilde{w}_{e}-r_{d} \widetilde{w}_{e}+r_{d} \widetilde{w}_{c}+\alpha_{d} r_{d} D \tilde{v}=0 \\
& \widetilde{w}_{a}+r_{d} \widetilde{w}_{e}+r_{c} D^{2} \widetilde{w}_{c}-\widetilde{w}_{c}-r_{d} \widetilde{w}_{c}-\left(\alpha_{b}+r_{d} \alpha_{d}\right) D \tilde{v}=0 \\
& \alpha_{b} D \tilde{w}_{a}+\alpha_{d} r_{d} D \tilde{w}_{e}-\left(\alpha_{b}+\alpha_{d} r_{d}\right) D \tilde{w}_{c}+r_{f} D^{4} \bar{v}-\left(\alpha_{b}^{2}+r_{d} \alpha_{d}^{2}\right) D^{2} \tilde{v}=0
\end{aligned}
$$

in which $D$ denotes the differential operator. From Eq. (19)a-d, by adding equations (19)a, b to Eq. (19)c, one obtains

$$
\begin{aligned}
& r_{a} D^{2} \widetilde{w}_{a}-\widetilde{w}_{a}+\widetilde{w}_{c}+\alpha_{b} D \tilde{v}=0 \\
& r_{e} D^{2} \widetilde{w}_{e}-r_{d} \widetilde{w}_{e}+r_{d} \tilde{w}_{c}+\alpha_{d} r_{d} D \tilde{v}=0 \\
& r_{a} D^{2} \widetilde{w}_{a}+r_{e} D^{2} \widetilde{w}_{e}+r_{c} D^{2} \widetilde{w}_{c}=0 \\
& \alpha_{b} D \widetilde{w}_{a}+\alpha_{d} r_{d} D \tilde{w}_{e}-\left(\alpha_{b}+\alpha_{d} r_{d}\right) D \tilde{w}_{c}+r_{f} D^{4} \bar{v}-\left(\alpha_{b}^{2}+r_{d} \alpha_{d}^{2}\right) D^{2} \tilde{v}=0
\end{aligned}
$$

From Eqs. (20)a-b, one has

$$
\begin{aligned}
& \widetilde{w}_{a}=r_{a} D^{2} \widetilde{w}_{a}+\widetilde{w}_{c}+\alpha_{b} D \tilde{v} \\
& \widetilde{w}_{e}=\frac{r_{e}}{r_{d}} D^{2} \widetilde{w}_{e}+\widetilde{w}_{c}+\alpha_{d} D \tilde{v}
\end{aligned}
$$

and from Eqs.(20)c-d, by taking the derivative of Eq. (20)d with respect to $\xi$, one obtains 


$$
\begin{aligned}
& D^{2} \widetilde{w}_{a}=\frac{\alpha_{b} r_{e}+\alpha_{d} r_{d} r_{e}+\alpha_{d} r_{c} r_{d}}{\alpha_{b} r_{e}-\alpha_{d} r_{a} r_{d}} D^{2} \widetilde{w}_{c}-\frac{r_{e} r_{f}}{\alpha_{b} r_{e}-\alpha_{d} r_{a} r_{d}} D^{5} \tilde{v}+\frac{\alpha_{b}^{2} r_{e}+\alpha_{d}^{2} r_{d} r_{e}}{\alpha_{b} r_{e}-\alpha_{d} r_{a} r_{d}} D^{3} \tilde{v} \\
& D^{2} \widetilde{w}_{e}=-\frac{\alpha_{b} r_{c}+\alpha_{b} r_{a}+\alpha_{d} r_{a} r_{d}}{\alpha_{b} r_{e}-\alpha_{d} r_{a} r_{d}} D^{2} \widetilde{w}_{c}+\frac{r_{a} r_{f}}{\alpha_{b} r_{e}-\alpha_{d} r_{a} r_{d}} D^{5} \tilde{v}-\left(\frac{\alpha_{b}^{2} r_{a}+\alpha_{d}^{2} r_{a} r_{d}}{\alpha_{b} r_{e}-\alpha_{d} r_{a} r_{d}}\right) D^{3} \tilde{v}
\end{aligned}
$$

From Eqs. (22)a-b and (21)a-b, by eliminating $\widetilde{w}_{a}$ and $\widetilde{w}_{e}$, one obtains

$$
\begin{aligned}
& \left(\alpha_{b} r_{e}+\alpha_{d} r_{d} r_{e}+\alpha_{d} r_{c} r_{d}\right) r_{a} D^{4} \tilde{w}_{c}-\left(r_{a}+r_{e}+r_{c}\right) r_{d} \alpha_{d} D^{2} \widetilde{w}_{c} \\
& =r_{a} r_{e} r_{f} D^{7} \tilde{v}-\left(r_{f}+\alpha_{b}^{2} r_{a}+\alpha_{d}^{2} r_{a} r_{d}\right) r_{e} D^{5} \tilde{v}+\left(\alpha_{d} r_{e}+\alpha_{b} r_{a}\right) r_{d} \alpha_{d} D^{3} \tilde{v} \\
& \left(\alpha_{b} r_{c}+\alpha_{b} r_{a}+\alpha_{d} r_{a} r_{d}\right) r_{e} D^{4} \widetilde{w}_{c}-\left(r_{a}+r_{e}+r_{c}\right) r_{d} \alpha_{b} D^{2} \widetilde{w}_{c} \\
& =r_{a} r_{e} r_{f} D^{7} \tilde{v}-\left(r_{d} r_{f}+\alpha_{b}^{2} r_{e}+\alpha_{d}^{2} r_{d} r_{e}\right) r_{a} D^{5} \tilde{v}+\left(\alpha_{b} r_{a}+\alpha_{d} r_{e}\right) r_{d} \alpha_{b} D^{3} \tilde{v}
\end{aligned}
$$

From Equations (23)a-b, by solving for fourth and second order derivatives of $\bar{w}_{c}$ and eliminating $\bar{w}_{c}$, one obtains an equation of only transverse displacement $\tilde{v}$. By assuming that the solution of displacement $\tilde{v}$ takes an exponential form $\tilde{v}=C e^{m \xi}$ and substituting into the resulting equation, one obtains

$$
m^{5}\left(A m^{4}+B m^{2}+C\right)=0
$$

Equation (24) has five zero roots and four non-zero inter-different roots, by solving the differential equation, the closed form solution for $\tilde{v}$ can be obtained as

$$
\tilde{v}=C_{1}+C_{2} \xi+C_{3} \xi^{2}+C_{4} \xi^{3}+C_{5} \xi^{4}+\sum_{k=6}^{9} C_{k} e^{m_{k} \xi}
$$

where $C_{1}, \cdots, C_{9}$ are unknown integration constants and $m_{k}(k=6, \ldots, 9)$ are non-zero distinct roots of Eq. (24). The integration can be determined based on boundary conditions. From Eq.(25), the closed form solutions for $\widetilde{w}_{c}, \widetilde{w}_{a}, \widetilde{w}_{e}$ can be also obtained based on Eqs. (23)a-b and (21)a-b as 


$$
\begin{aligned}
& \tilde{v}=C_{1}+C_{2} \xi+C_{3} \xi^{2}+C_{4} \xi^{3}+C_{5} \xi^{4}+\sum_{k=6}^{9} C_{k} e^{m_{k} \xi} \\
& \tilde{w}_{c}=-3 L_{1} C_{4} \xi^{2}-4 L_{1} \xi^{3} C_{5}+\sum_{k=6}^{9} R_{k} e^{m_{k} \xi} C_{t}+C_{10}+C_{11} \xi \\
& \tilde{w}_{a}=\alpha_{b} C_{2}+2 \alpha_{b} \xi C_{3}+3 L_{2}\left(2 r_{a}+\xi^{2}\right) C_{4}+4 L_{2}\left(6 r_{a} \xi+\xi^{3}\right) C_{5}+\sum_{k=6}^{9} S_{k} e^{m_{k} \xi} C_{t}+C_{10}+C_{11} \xi \\
& \tilde{w}_{e}=\alpha_{d} C_{2}+2 \alpha_{d} \xi C_{3}+3 L_{3}\left(2 \frac{r_{e}}{r_{d}}+\xi^{2}\right) C_{4}+4 L_{3}\left(6 \frac{r_{e}}{r_{d}} \xi+\xi^{3}\right) C_{5}+\sum_{k=6}^{9} T_{k} e^{m_{k} \xi} C_{t}+C_{10}+C_{11} \xi
\end{aligned}
$$

in which

$$
\begin{aligned}
& L_{1}=\frac{\left(\alpha_{b} r_{a}+\alpha_{d} r_{e}\right)}{\left(r_{a}+r_{e}+r_{c}\right)} ; \quad L_{2}=\frac{\left(\alpha_{b} r_{e}-\alpha_{d} r_{e}+\alpha_{b} r_{c}\right)}{\left(r_{a}+r_{e}+r_{c}\right)} ; \quad L_{3}=\frac{\left(-\alpha_{b} r_{a}+\alpha_{d} r_{a}+\alpha_{d} r_{c}\right)}{\left(r_{a}+r_{e}+r_{c}\right)} ; \\
& R_{k}=\frac{r_{a} r_{c} r_{e} r_{f}\left(\alpha_{b} r_{e}-\alpha_{d} r_{a} r_{d}\right)}{\left\{\alpha_{b} \alpha_{d} r_{c}\left(r_{a} r_{d}-r_{e}\right)+r_{a} r_{e}\left(\alpha_{b}-\alpha_{d}\right)\left(\alpha_{b}+\alpha_{d} r_{d}\right)\right\} r_{d}\left(r_{a}+r_{e}+r_{c}\right)} m_{k}^{5}-\frac{\left(\alpha_{b} r_{a}+\alpha_{d} r_{e}\right)}{\left(r_{a}+r_{e}+r_{c}\right)} m_{k} \\
& +\frac{\left(\alpha_{d} r_{a}^{2} r_{d}^{2}-\alpha_{b} r_{e}^{2}\right) r_{c} r_{f}+\left(\alpha_{b}+\alpha_{d} r_{d}\right)\left(r_{d} r_{a}-r_{e}\right) r_{a} r_{e} r_{f}+\left(\alpha_{d} r_{a} r_{d}-\alpha_{b} r_{e}\right)\left(\alpha_{b}^{2}+\alpha_{d}^{2} r_{d}\right) r_{a} r_{c} r_{e}}{\left\{\alpha_{b} \alpha_{d} r_{c}\left(r_{a} r_{d}-r_{e}\right)+r_{a} r_{e}\left(\alpha_{b}-\alpha_{d}\right)\left(\alpha_{b}+\alpha_{d} r_{d}\right) r_{d}\left(r_{a}+r_{e}+r_{c}\right)\right.} m_{k}^{3} ; \\
& S_{k}=R_{k}+\alpha_{b} m_{k}+\frac{r_{a}\left(\alpha_{b} r_{e}+\alpha_{d} r_{d} r_{e}+\alpha_{d} r_{c} r_{d}\right)}{\left(\alpha_{b} r_{e}-\alpha_{d} r_{a} r_{d}\right)} R_{k} m_{k}^{2}+\frac{\left(\alpha_{b}^{2} r_{a} r_{e}+\alpha_{d}^{2} r_{a} r_{d} r_{e}\right)}{\left(\alpha_{b} r_{e}-\alpha_{d} r_{a} r_{d}\right)} m_{k}^{3}-\frac{r_{a} r_{e} r_{f}}{\left(\alpha_{b} r_{e}-\alpha_{d} r_{a} r_{d}\right)} m_{k}^{5} ; \\
& T_{k}=R_{k}+\alpha_{d} m_{k}-\frac{\alpha_{b} r_{c} r_{e}+\alpha_{b} r_{a} r_{e}+\alpha_{d} r_{a} r_{d} r_{e}}{r_{d}\left(\alpha_{b} r_{e}-\alpha_{d} r_{a} r_{d}\right)} R_{k} m_{k}^{2}-\frac{\alpha_{b}^{2} r_{a} r_{e}+\alpha_{d}^{2} r_{a} r_{d} r_{e}}{r_{d}\left(\alpha_{b} r_{e}-\alpha_{d} r_{a} r_{d}\right)} m_{k}^{3}+\frac{r_{a} r_{e} r_{f}}{r_{d}\left(\alpha_{b} r_{e}-\alpha_{d} r_{a} r_{d}\right)} m_{k}^{5} ;
\end{aligned}
$$

Equations (26) involve 11 unknown integration constants $C_{1}, \ldots, C_{11}$ while we have only ten boundary conditions. This is a result of the fact that, during the solution procedure, we have taken a derivative of Eq. (20)-d with respect to $\xi$, i.e., Eq.(22)-b. Therefore, from Eqs. (26), by substituting into Eq. (20)-d, it can be shown that constant $C_{5}$ vanishes. By setting constants $C_{1}, C_{2}, C_{3}, C_{4}$ equal to $D_{1}, D_{2}, D_{3}, D_{4}$, and $C_{6}, C_{7}, C_{8}, C_{9}, C_{10}, C_{11}$ equal to $D_{5}, D_{6}, D_{7}, D_{8}, D_{9}, D_{10}$, respectively, on recovers the solution in Eqs. (14). 


\section{REFERENCES}

Accord, N.B. and Earls, C. J. (2006), Use of Fiber-Reinforced Polymer Composite Elements to Enhance Structural Steel Member Ductility, Journal of Composites for Construction, 10(4), 337344.

Aguilera, J. and Fam, A. (2013), Bonded FRP Plates for Strengthening Rectangular Hollow Steel Section T-Joints against Web Buckling Induced by Transverse Compression, Journal of Composites for Construction, ASCE, 17(4), 421-432.

Asik, M.Z. and Tezcan, S. (2005), A mathematical model for the behavior of laminated glass beams, Computers and Structures, 83 (21-22), 1742-1753.

Bonacci, J.F. and Maalej, M. (2000), Externally bonded fiber-reinforced polymer for rehabilitation of corrosion damaged concrete beams, ACI Structural Journal, 97(5), 703-711.

Correia, J.R., Branco, F.A., Silva, N.M.F, Camotim, D. and Silvestre, N. (2011), First-order, buckling and post-buckling behaviour of GFRP pultruded beams. Part 1: Experimental study, Computers and Structures, 89(21-22), 2052-2064.

Dawood, M.M.R., (2008), Bond characteristics and environmental durability of CFRP materials for strengthening steel bridges and structures, Ph.D. thesis, North Carolina State University. Elchalakani, M. and Fernando, D. (2012), Plastic mechanism analysis of unstiffened steel I-section beams strengthened with CFRP under 3-point bending, Thin-Walled Structures, 53, 58-71.

El Damatty, A.A. and Abushagur, M. (2003), Testing and modeling of shear and peel behavior for bonded steel/FRP connections, Thin-Walled Structures, 41(11), 987-1003

El Damatty, A., Abushagur, M. and Youssef, M. A. (2003), Experimental and analytical investigation of steel beams rehabilitated using GFRP sheets, Journal of Steel \& Composite Structures 3(6), 421-438. 
Fam, A., MacDougall and Shaat, A. (2009) "Upgrading Steel-Concrete Composite Girders and Repair of Damaged Steel Beams using Bonded CFRP Laminates", Thin-Walled Structures, 47(10):1122-1135.

Ganga, H.V.S., Taly, N., and Vijay, P.V., (2007), Reinforce Concrete Design, Taylor and Francis Group publisher.

Gjelsvik A. (1981), The Theory of Thin Walled Bars, $1^{\text {st }}$ Edition, New York, et al., John Wiley \& Sons publishing Incorporation.

Ghafoori, E, Motavalli, M., (2013) Flexural and interfacial behavior of metallic beams strengthened by prestressed bonded plates, Journal of composite structures, 101, 22-34.

Kim, H.,S. and Shi, S., Y., (2011), Flexural behaviour of reinforced concrete (RC) beams retrofitted with hybrid fiber reinforced polymers (FRPs) under sustaining loads, Journal of Composite Structures, 93(2), 802-811.

Hall, J., (2002), Adhesives-Section B15 Kempe's Engineers Year-Book, 2000. Adhesive Design Toolkit: http://www.adhesivestoolkit.com/Toolkits/DesignGuidance/AdhesiveType.xtp.

Harries, A. K. and El-Tawil, S. (2008) Steel-FRP composite structural systems. " Int. Conf. on Composite Construction in Steel and Concrete 2008, Composite Construction in Steel and Concrete VI, ASCE, Reston, VA, $703-716$

Hollaway, L.C., Zhang, L., Photiou, N.K., Teng, J.G. and Zhang, S.S. (2006). Advances in Adhesive Joining of Carbon Fiber/Polymer Composites to Steel Members for Repair and Rehabilitation of Bridge Structures. Advances in Structural Engineering, 9(6), 791-803.

Huveners, E.M.P, Gerwijnen, F.V., Soetens, F., Hofmeyer, H. (2007), Mechanical shear properties of adhesives, Proceedings of the 10th International Conference on Architectural and Automotive Glass, 15-18 June 2007, Tampere, Finland: Glass Performance Days, Tamglass Ltd. Oy, 367-370. 
Narmashiri, K., Ramli Sulong, N.H. and Jumaat, M.Z. (2012), Failure analysis and structural behaviour of CFRP strengthened steel I-beams, Construction and Building Materials, Volume 30, May 2012, Pages $1-9$.

Mallick, P., K., (1988), Fiber Reinforced Composites: Materials, Manufacturing, and Design, Third Edition, Taylor and Francis Group publisher.

Miller, C.T., Chajes J.M. and Hastings N.J. (2001), Strengthening of a steel bridge girder using CFRP plates, Journal of Bridge Engineering 6(6), 514-522.

Linghoff, D. and Al-Emrani, M. (2010), Performance of steel beams strengthened with CFRP laminate - Part 2: Laboratory tests, Composites Part B, 41(7), 516-522

Linghoff, D., Al-Emrani and Kliger, M. R. (2010), Performance of steel beams strengthened with CFRP laminate - Part 1: Laboratory tests, Composites Part B, 41(7), 509-515.

Liu, P., Zhao, Q., Li, F, Liu, J, Chen, H., (2014), Research on the Mechanical Properties of a Glass Fiber Reinforced Polymer-Steel Combined Truss Structure, The Scientific World Journal, volume 2014, Article ID 309872,

Liu, Y. and Gannon, L., (2009), Finite element study of steel beams reinforced while under load, Journal of Engineering Structures, 31(11), 2630-2642.

Pham, P.V., (2013), Stress-Deformation Theories for the Analysis of Steel Beams Reinforced with GFRP Plates, Master of Science Thesis, Department of Civil Engineering, University of Ottawa, Ottawa, ON, Canada.

Pham, P.V. and Mohareb, M. (2014), A shear deformable theory for the analysis of steel beams reinforced with GFRP plates, Thin-Walled Structures, 85, 165-182. 
Pham, P.V. and Mohareb, M. (2015a), Finite-Element Formulations for the Spatial Static Response of Steel Beams Bonded to a GFRP Plate, Journal of Engineering Mechanics, ASCE, 141(4), 04014143.

Pham, P.V and Mohareb, M. (2015b), Nonshear Deformable Theory for Analysis of Steel Beams Reinforced with GFRP Plate Closed-Form Solution, Journal of Structural Engineering, ASCE, 141(12), 04015063.

Richardson, T. and Fam, A. (2014) "Modulus Effect of Bonded CFRP Laminates used for Repairing Pre- and Post-Yield Cracked Concrete Beams", ASCE Journal of Composites for Construction, 18(4):04013054.

Sahin, M., U. and Dawood, M., (2016), Experimental investigation of bond between HighModulus CFRP and Steel at moderately elevated temperatures, Journal of composites for constructions, ASCE, 10.1061/ (ASCE) CC.1943-5614.0000702, 04016049.

Shaat, A., and Fam, A. (2009), Slender steel columns strengthened using high modulus CFRP plates for buckling control, Journal of Composites for construction, 13(1), 2-12.

Siddique, M.A.A. and El Damatty, A.A. (2012), Enhancement of buckling capacity of steel plates strengthened with GFRP plates, Thin-Walled Structures, 60(16), 154-162

Siddique, M.A.A. and El Damatty, A.A. (2013), Improvement of local buckling behaviour of steel beams through bonding GFRP plates, Composite Structures, 96(6), 44-56

Quin, W. Y., Liang, Z., Xiang, Z.R., Yue, L.X., and Jiu, S.Y., (2015), Behaviour of I-section steel beam welding reinforced while under load, Journal of Constructional Steel Research, 106, 278288.

Teng, J.G., and Hu, Y.M. (2007). Behaviour of FRP-jacketed circular steel tubes and cylindrical shells under axial compression. Construction and Building Materials, 21(4), 827-838. 
Tomblin, J., Seneviratne, W., Escobar, P., and Khian, Y., Y., (2002), Shear stress-strain data for structural adhesives, Technical Report, National Technical Information Service (NTIS), Springfield, Virginia, 22161.

Torabizadeh, M.A., (2013), Tensile, compressive and shear properties of unidirectional glass/epoxy composites subjected to mechanical loading and low temperature services, Indian Journal of Engineering \& Materials Sciences, 20(4), 299-309.

Wenwei, W. and Guo, L.(2006), Experimental study and analysis of RC beams strengthened with CFRP laminates under sustaining load, Internal journal of solids and structures, 43(6), 1372-1387. Westover, C.M., (1998), The compressive behaviour of Glass Reinforced Composite subjected to local Thermal Loading, Master thesis of Engineering, University of California, Berkeley.

Wu, Z., Shao, Y., Iwashita, K., and Sakamoto, K., (2007), Strengthening of Preloaded RC Beams using Hybrid Carbon Sheets, Journal of composites for construction, ASCE, 11(3), 299-307.

Youssef, M. (2006). Analytical Prediction of the Linear and Nonlinear Behaviour of Steel Beams Rehabilitated using FRP Sheets, Engineering Structures, 28(6), 903-911.

Zhao, X.-L. and Zhang, L. (2007), State-of-the-art review on FRP strengthened steel structures, Engineering Structures, 29(8), 1808-1823. 
Table 1. Summary of loads and displacements in configurations 1-6

\begin{tabular}{|c|c|c|c|c|c|c|c|}
\hline \multirow{2}{*}{ Fields } & \multirow{2}{*}{ Component } & \multicolumn{6}{|c|}{ Configuration } \\
\hline & & 1 & 2 & 3 & 4 & 5 & 6 \\
\hline \multirow{4}{*}{ Total Loads } & GFRP plate a & - & - & 0 & $q_{a, 4}$ & 0 & 0 \\
\hline & Steel beam c & $q_{c, 1}=0$ & $q_{c, 2}$ & $q_{c, 3}=q_{c, 2}$ & $q_{c, 4}=q_{c, 2}$ & $q_{c, 5}=q_{c, 2}$ & $q_{c, 2}+q_{c, 6}$ \\
\hline & GFRP plate $\mathrm{e}$ & - & - & 0 & $q_{e, 4}$ & 0 & 0 \\
\hline & Sum & 0 & $q_{c, 2}$ & $q_{c, 2}$ & $q_{a, 4}+q_{c, 2}+q_{e, 4}$ & $q_{c, 2}$ & $q_{c, 2}+q_{c, 6}$ \\
\hline \multirow{3}{*}{$\begin{array}{l}\text { Total Transverse } \\
\text { Displacements } \\
\text { (measured relative to } \\
\text { configuration 1) }\end{array}$} & GFRP plate a & - & - & 0 & \multirow{3}{*}{$v_{4}=v_{2}$} & \multirow{3}{*}{$v_{5}$} & \multirow{3}{*}{$v_{6}$} \\
\hline & Steel beam c & 0 & $v_{2}$ & $v_{3}=v_{2}$ & & & \\
\hline & GFRP plate $\mathrm{e}$ & - & - & 0 & & & \\
\hline \multirow{3}{*}{$\begin{array}{l}\text { Total Longitudinal } \\
\text { Displacement (measured } \\
\text { relative to configuration } \\
\text { (1) for steel beam or } \\
\text { relative to configuration } \\
\text { (4) for GFRP plates) }\end{array}$} & GFRP plate a & - & - & 0 & 0 & $w_{a, 5}$ & $w_{a, 6}$ \\
\hline & Steel beam c & 0 & 0 & 0 & 0 & $w_{c, 5}$ & $w_{c, 6}$ \\
\hline & GFRP plate e & - & - & 0 & 0 & $w_{e, 5}$ & $w_{e, 6}$ \\
\hline
\end{tabular}

Table 2. Maximum and minimum longitudinal normal stresses (MPa)

\begin{tabular}{|c|c|c|c|c|c|c|c|c|c|c|c|c|c|}
\hline \multirow{3}{*}{$\begin{array}{c}t_{a} \\
(\mathrm{~mm})\end{array}$} & \multirow{3}{*}{$\begin{array}{c}t_{e} \\
(\mathrm{~mm})\end{array}$} & \multicolumn{6}{|c|}{ Wide Flange Steel Beam } & \multicolumn{6}{|c|}{ GFRP plate } \\
\hline & & \multicolumn{3}{|c|}{ Compression } & \multicolumn{3}{|c|}{ Tension } & \multicolumn{3}{|c|}{$a$} & \multicolumn{3}{|c|}{$e$} \\
\hline & & $\begin{array}{l}\text { Present } \\
\text { Study }\end{array}$ & $\begin{array}{l}\text { 3D- } \\
\text { FEA }\end{array}$ & $\begin{array}{c}\% \\
\text { Diff.* }\end{array}$ & $\begin{array}{l}\text { Present } \\
\text { Study }\end{array}$ & $\begin{array}{l}\text { 3D- } \\
\text { FEA }\end{array}$ & $\begin{array}{c}\% \% \\
\text { Diff. }\end{array}$ & $\begin{array}{l}\text { Present } \\
\text { Study }\end{array}$ & $\begin{array}{l}\text { 3D- } \\
\text { FEA }\end{array}$ & $\begin{array}{c}\% \\
\text { Diff. }\end{array}$ & $\begin{array}{l}\text { Present } \\
\text { Study }\end{array}$ & $\begin{array}{l}\text { 3D- } \\
\text { FEA }\end{array}$ & $\begin{array}{c}\% \\
\text { Diff. }\end{array}$ \\
\hline 9 & 29 & -137 & -138 & 0.3 & 119 & 119 & 0.0 & -13.5 & -13.4 & 0.7 & 14.2 & 14.3 & 0.7 \\
\hline 19 & 19 & -127 & -127 & 0.0 & 127 & 127 & 0.0 & -13.7 & -13.5 & 1.0 & 13.7 & 13.5 & 1.1 \\
\hline 29 & 9 & -118 & -118 & 0.4 & 137 & 138 & 0.3 & -14.2 & -14.4 & 1.0 & 13.3 & 13.4 & 1.1 \\
\hline
\end{tabular}

$* \%$ difference $=(3$ DFEA - Present study $) * 100 / 3$ DFEA

Table 3. Comparison of results based on 3D FEA, present study and transformed section

\begin{tabular}{|c|c|c|c|c|c|c|c|}
\hline \multirow{3}{*}{ Solution } & \multirow{2}{*}{$\begin{array}{c}\text { Gb, Gd } \\
(\mathrm{MPa})\end{array}$} & \multicolumn{2}{|c|}{$\begin{array}{c}\text { Peak deflection } \\
(\mathrm{mm})\end{array}$} & \multicolumn{2}{c|}{$\begin{array}{c}\text { Normal stress in Steel } \\
(\mathrm{MPa})\end{array}$} & \multicolumn{2}{c|}{$\begin{array}{c}\text { Normal stress in GFRP } \\
(\mathrm{MPa})\end{array}$} \\
\cline { 3 - 8 } & & Value & \% difference & Value & \% difference & Value & $\%$ difference \\
\hline \multirow{3}{*}{$\begin{array}{c}\text { Present } \\
\text { study }\end{array}$} & 1300 & 8.1 & 2.4 & 127 & 0.0 & 13.5 & 0.1 \\
\cline { 2 - 8 } & 400 & 8.1 & 2.4 & 127 & 0.0 & 13.5 & 0.1 \\
\cline { 2 - 8 } & 1.3 & 8.9 & 2.2 & 139 & 0.7 & 10.8 & 0.0 \\
\hline \multirow{3}{*}{$\boldsymbol{T S}^{1}$} & 1300 & $\mathbf{8 . 0}$ & 1.2 & $\mathbf{1 2 6 . 5}$ & 0.4 & $\mathbf{1 1 . 4}$ & 15.5 \\
\cline { 2 - 8 } & 400 & $\mathbf{8 . 0}$ & 1.2 & $\mathbf{1 2 6 . 5}$ & 0.4 & $\mathbf{1 1 . 4}$ & 15.5 \\
\cline { 2 - 8 } & 1.3 & $\mathbf{8 . 0}$ & 10.1 & $\mathbf{1 2 6 . 5}$ & 9.0 & $\mathbf{1 1 . 4}$ & 5.6 \\
\hline \multirow{3}{*}{ 3D FEA } & 1300 & 8.3 & 0.0 & 127 & 0.0 & 13.5 & 0.0 \\
\cline { 2 - 8 } & 400 & 8.3 & 0.0 & 127 & 0.0 & 13.5 & 0.0 \\
\cline { 2 - 8 } & 1.3 & 9.1 & 0.0 & 138 & 0.0 & 1.08 & 0.0 \\
\hline
\end{tabular}

1 TS = Transformed Section Method.

$2 \%$ difference $=($ present study or TS -3 D FEA $) \times 100 / 3$ D FEA; 